\title{
Density depletion profile and solvation free energy of a colloidal particle in a polymer solution
}

\author{
R. Maassen, E. Eisenriegler, and A. Bringer \\ Institut für Festkörperforschung, Forschungszentrum Jülich, D-52425 Jülich, Germany
}

(Received 5 April 2001; accepted 22 June 2001)

\begin{abstract}
The solvation free energy and polymer density depletion profile of a single mesoscopic colloidal particle in a solution of free nonadsorbing polymer chains are investigated theoretically. Keeping both the particle to polymer size ratio and the degree of inter-chain overlap arbitrary, we see how the qualitatively different behavior evolves in the limits of small and large size ratios and of dilute and semidilute solutions. While most of our results are obtained within a mean-field approach, we also use a "renormalized tree approximation" to estimate the surface tension and the coefficient of spontaneous curvature in a Helfrich expansion for large particle to polymer size ratio. There is a weak maximum in the polymer density profile for arbitrary size ratio. For small size ratio the maximum can be explained in terms of a minimum in the bulk polymer density correlation function.

(C) 2001 American Institute of Physics. [DOI: 10.1063/1.1394206]
\end{abstract}

\section{INTRODUCTION}

There is an effective interaction between colloidal particles in a solvent which contains nonadsorbing free polymer chains. Since the chains avoid the space between two close particles, the unbalanced polymer pressure from outside pushes the two particles toward each other. This depletion interaction is believed to be important for a variety of interesting colloids such as casein micelles, ${ }^{1}$ red blood cells, ${ }^{2}$ and globular proteins. ${ }^{3}$ It is an example of what is termed "macromolecular crowding" in the biophysical chemistry literature. ${ }^{4,5}$

The depletion of long flexible polymers near the surface of a colloidal particle is an entropic effect and depends in a crucial way on the ratio of the particle and chain sizes and on the degree of overlap between the chains, i.e., on whether the polymer solution is dilute or semidilute. The simplest system for studying both effects is a single spherical particle or a single cylindrical rod with radius $R$ embedded in a monodisperse solution of free nonadsorbing polymer chains.

One may characterize the degree of overlap between chains by $n / n^{*}$, where $n$ is the number density of chains in the bulk and $n^{*}$ is the density at the onset of overlap, ${ }^{6}$ and the size ratio by

$$
\rho=R / \mathcal{R}_{x} .
$$

Here $d \mathcal{R}_{x}^{2}$ is the mean square end-to-end distance of a single polymer chain in dilute solution without particles, and $d$ denotes the spatial dimension. Figure 1 shows various limits of a single spherical or cylindrical particle in a polymer solution.

One important limit is a planar wall, which can be considered as a particle with infinite radius $R$, for which $1 / \rho$ vanishes (upper and lower left corners in Fig. 1). The corresponding bulk-normalized polymer density profile $\mathcal{M}$ $=\mathcal{M}_{\mathrm{pw}}$ depends in a crucial way on the inter-chain overlap. In the semidilute limit $n \gg n^{*}$ it reaches its bulk value 1 for distances $z$ from the wall which are of the order of the screening length ${ }^{6-8}$ (or mesh-size) $\xi$. In the dilute limit $n$ $\ll n^{*}$ the corresponding "healing length" for $\mathcal{M}_{\mathrm{pw}}$ is ${ }^{6,9}$ of the order of the end-to-end distance $\propto \mathcal{R}_{x}$, introduced below Eq. (1.1).

The behavior is quite different in the opposite limit of a sphere or an infinitely long rod with small radius $R$, i.e., for $1 / \rho \rightarrow \infty$ (upper and lower right corners in Fig. 1), corresponding to $R \ll \xi, \mathcal{R}_{x}$. In this case the healing length of $\mathcal{M}=\mathcal{M}_{\mathrm{s}}$ is of the order ${ }^{10}$ of $R$. For distances $r$ from the center of the sphere (or $r_{\perp}$ from the axis of the cylinder) which are much smaller than $\xi$ or $\mathcal{R}_{x}$, the normalized profile $\mathcal{M}_{\mathrm{s}}$ is independent of the overlap and of $\xi, \mathcal{R}_{x}$ and only depends ${ }^{10-14}$ on $r / R$ (or $r_{\perp} / R$ ).

The free energy $F$ it costs to immerse the spherical or cylindrical particle in the polymer solution also shows qualitative differences ${ }^{15-18}$ in the various limits in Fig. 1.

In this paper we study the crossover between these limits and evaluate the density profile $\mathcal{M}(r)$ and the free energy cost $F$ of the single particle for arbitrary values of the size ratio and the inter-chain overlap.

An important relation between the two basic physical quantities $\mathcal{M}$ and $F$ which applies for arbitrary size ratio and overlap is the so-called density-pressure identity; see Refs. 14, 19, 20 and Appendix A. For example, for a cylinder of infinite length $\lambda \rightarrow \infty$ it relates the polymer pressure,

$$
p=\frac{1}{S_{\perp}} \frac{d}{d R} \frac{F}{\lambda},
$$

on the surface of the cylinder with surface area $S_{\perp} \lambda$ to the behavior $\mathcal{M}^{\text {as }}\left(r_{\perp}\right)$ of the normalized polymer density profile $\mathcal{M}\left(r_{\perp}\right)$ near the surface ${ }^{21}$ via

$$
\frac{n \mathcal{R}_{x}^{1 / \nu} \mathcal{M}^{(\text {as })}\left(r_{\perp}\right)}{\left(r_{\perp}-R\right)^{1 / \nu}}=B \frac{p}{k_{B} T} .
$$

Here $S_{\perp}=2 \pi R$ (or $S_{\perp}=4 \pi R^{2}$ ) is the circumference of the circle (or the surface area of the sphere) of radius $R$ of the cross-section perpendicular to the axis of the cylinder in 


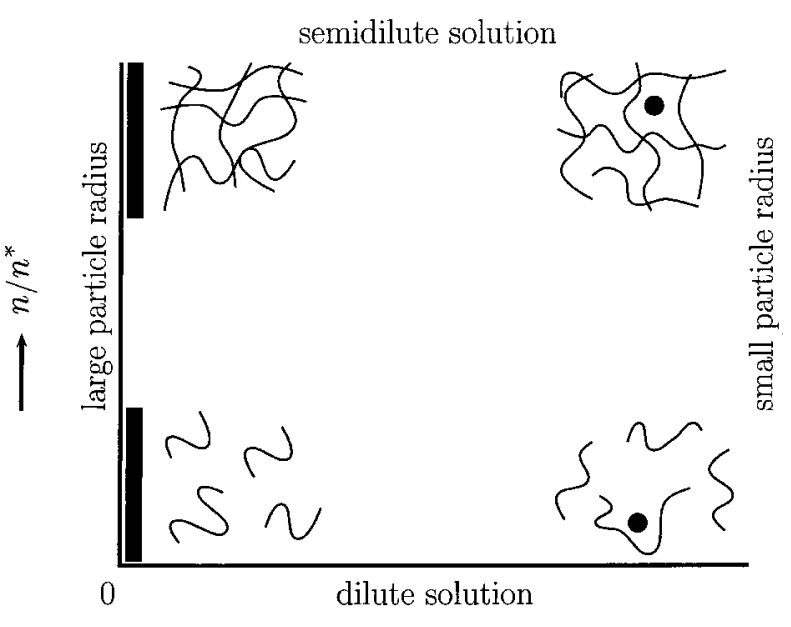

$\longrightarrow \mathcal{R}_{x} / R$

FIG. 1. Various limits of a single spherical particle or a single cylindrical rod in a solution of nonadsorbing polymers. The sphere or rod becomes a planar wall for vanishing $\mathcal{R}_{x} / R$ (i.e., for points on the vertical axis), and becomes a "small" sphere or a "thin" rod with a radius much smaller than the characteristic polymer lengths (such as the root mean square end-to-end distance $\propto \mathcal{R}_{x}$ in the dilute solution or the mesh-size $\xi$ in the semidilute solution) as $\mathcal{R}_{x} / R$ becomes large with the inter-chain overlap $n / n *$ kept fixed. The following limits are shown: planar wall in a dilute solution (lower left corner), planar wall in a semidilute solution (upper left corner), small sphere or a thin rod in a dilute solution (lower right corner), and small sphere or a thin rod in a semidilute solution (upper right corner).

three (or four ${ }^{22}$ ) dimensions, $r_{\perp}$ is the distance of $\mathbf{r}$ from the axis, $\nu$ is the Flory exponent $\mathrm{e}^{6-8}$ and $B$ is a universal amplitude. ${ }^{20,23}$ The denominator $\left(r_{\perp}-R\right)^{1 / \nu}$ on the left-hand side cancels the $r_{\perp}$-dependence ${ }^{21}$ of $\mathcal{M}^{\text {(as) }}$, and both sides in Eq. (1.3) only depend on $R, \mathcal{R}_{x}$, and $n$. The identity is free of microscopic parameters and no proportionality factors have been omitted.

The universal properties ${ }^{6-9}$ of long flexible polymers in a good solvent that interact with an embedded nonadsorbing mesoscopic colloidal particle can be calculated from a simple model in which each polymer molecule is represented by a "spring and bead" chain. Each bead is pointlike and is excluded from the space occupied by the particle, and beads of the same chain or of different chains repel each other at microscopic distances. Despite the simplicity of the model the conformational statistics of the polymers for arbitrary size ratio and overlap is quite complex, and one has to resort to approximations, as in integral-equation based approaches. $^{24}$ In earlier work of this type a linear ${ }^{25}$ increase of $\mathcal{M}^{\text {(as) }}$ with distance $r_{\perp}-R$ or $r$-R from the particle surface was reported. However, Fuchs and Schweizer ${ }^{26}$ recently made an ansatz for the effective bead-particle repulsion with a mesoscopic range to describe the change of polymer conformations near the particle surface which implies a quadratic increase. This is consistent with a value $\nu=1 / 2$.

In this paper we calculate $\mathcal{M}$ and $F$ for arbitrary size ratio and inter-chain overlap for the dimension $d=4-\epsilon$ of the polymer-embedding space $^{22}$ close to the upper critical dimension $d=4$. We consider the leading order results for $\epsilon \searrow 0$ which are determined by the tree (or mean-field) approximation. This systematic approach leads in a transparent way to instructive results. In particular, the density-pressure identity follows without any further assumptions. The meanfield results are useful, since most of the qualitative features in $d$ near 4 presumably persist down to $d=3$. Moreover we will use mean-field scaling functions to construct for some of the observables a "renormalized tree approximation" which works directly in three dimensions.

For illustration we recall known analytical mean-field results valid for some of the limiting cases in Fig. 1 for an infinitely long cylinder (rod) in four dimensions. (i) For small radius $R(\rho \rightarrow 0)$ the normalized density profile and the free energy cost per unit axis length $\lambda$ are given by ${ }^{12,13}$

$$
\mathcal{M}=\left(1-\frac{R}{r_{\perp}}\right)^{2} ; \quad R, r_{\perp} \ll \mathcal{R}_{x}, \xi
$$

and

$$
\frac{F}{k_{B} T \lambda}=2 \pi n R \mathcal{R}_{x}^{2}
$$

These expressions apply for arbitrary overlap $n / n^{*}$. (ii) In the mean-field approximation a dilute polymer solution $\left(n / n^{*} \rightarrow 0\right)$ corresponds to a solution of ideal chains without excluded volume interaction between monomers. The free energy cost per unit axis length $\lambda$ for arbitrary size ratio $\rho$ is ${ }^{13,15}$

$$
\frac{F}{k_{B} T \lambda}=2 \pi n R \mathcal{R}_{x}^{2}\left(1+2 \sqrt{\frac{2}{\pi}} \rho+\frac{2}{3} \rho^{2}\right) .
$$

The profile $\mathcal{M}$, which is also known for this case, ${ }^{27}$ and the free energy cost Eq. (1.6) satisfy ${ }^{28}$ the density-pressure identity and reduce, for $\rho \rightarrow 0$, to the thin cylinder expressions in (i).

Analytical mean-field results for large radius (planar wall) are also available in the semidilute limit ${ }^{6,19}$ and will be mentioned in Sec. II E. No analytical mean-field results seem to be known for the dilute-semidilute crossover in the planar wall limit (vertical axis in Fig. 1) and for the crossover in size ratio in the semidilute limit (horizontal line for large $\left.n / n^{*}\right)$.

After the mean-field treatment in Sec. II, we discuss in Sec. III the overlap-dependence of the surface tension of a planar wall and of the coefficient of spontaneous curvature in a Helfrich expansion ${ }^{29}$ using a "renormalized tree approximation." Our results are summarized in Sec. IV. Technical details that can be skipped in a first reading are relegated to Appendices A-E.

\section{MEAN-FIELD APPROACH CLOSE TO FOUR DIMENSIONS}

\section{A. Epsilon expansion and mean-field equations}

The self-consistent mean-field approximation for polymer solutions also goes under the names of the randomphase or tree approximation. ${ }^{6,8}$ In this approximation the mean square end-to-end distance $d \mathcal{R}_{x}^{2}$ of a single chain with $N$ segments takes the ideal-chain form, where

$$
\mathcal{R}_{x}^{2}=2 N l^{2} \equiv 2 L,
$$


and $l$ is an effective segment size. The osmotic pressure $\Pi$ of a solution of overlapping chains takes the Flory-Huggins form

$$
\Pi /\left(k_{B} T\right)=n(1+\mathcal{S} / 2),
$$

with

$$
\mathcal{S}=b N^{2} n
$$

Here $b \delta\left(\mathbf{r}_{J, j}-\mathbf{r}_{K, k}\right)$ models the excluded volume interaction per $k_{B} T$ between monomer $j$ of chain $J$ and monomer $k$ of chain $K$.

In the following we consider a polymer-embedding space of dimension $d=4-\epsilon$. To leading order in the $\epsilon$-expansion the polymer solution displays mean-field behavior, with the interaction constant $b$ replaced by its fixed point value $7,8,22,30$

$$
b=b_{\mathrm{FP}} \equiv 2 \pi^{2} \epsilon l^{4},
$$

and the quantity $\mathcal{S}$ in Eqs. (2.2) and (2.3) is given by ${ }^{8,30}$

$$
\mathcal{S}=\epsilon \frac{\pi^{2}}{2} s_{4}^{(x)}=\frac{n}{n^{*}} .
$$

Here

$$
s_{d}^{(x)}=\left(\mathcal{R}_{x}\right)^{d} n
$$

characterizes the geometrical overlap between chains, and

$$
n^{*} \equiv\left(A_{2}\right)^{-1}=\left(\epsilon \frac{\pi^{2}}{2} \mathcal{R}_{x}^{4}\right)^{-1},
$$

where $A_{2}=b_{\mathrm{FP}} N^{2}$, the second virial coefficient of the osmotic pressure, is a convenient quantity marking the crossover between dilute $\left(n \ll n^{*}\right)$ and semidilute $\left(n \gg n^{*}\right)$ behavior. ${ }^{31}$ In $4-\epsilon$ dimensional space with small $\epsilon$, two polymer chains rarely cross, ${ }^{8}$ and the chain density in the crossover region $\left(n \approx n^{*}\right)$ corresponds to a very large geometrical overlap $s_{d}^{(x)}$ of order $1 / \epsilon$.

We now turn to the case of a polymer solution with an imbedded mesoscopic particle or wall, in which the monomer density $N n \mathcal{M}(\mathbf{r})$ becomes r-dependent. On replacing the excluded volume interaction by a configurationindependent external potential per $k_{B} T$ given by $b N n \mathcal{M}(\mathbf{r})$ and acting on each chain-monomer, the mean-field approximation reduces the many-chain problem to the problem of one ideal chain in a potential to be determined selfconsistently.

To calculate $\mathcal{M}(\mathbf{r})$ in Eq. (2.12), we need the partition function $Z\left(L^{\prime}, \mathbf{r}\right)$ of a chain with a polymerization index $N^{\prime}=L^{\prime} / l^{2}$ smaller than $N$ and with one end fixed at $\mathbf{r}$. This is determined by a diffusion-type equation ${ }^{6,32}$

$$
\left(\frac{\partial}{\partial L^{\prime}}-\Delta_{\mathbf{r}}+\mathcal{V}(\mathbf{r})\right) Z\left(L^{\prime}, \mathbf{r}\right)=0,
$$

where

$$
\mathcal{V}(\mathbf{r})=l^{-2} b N n \mathcal{M}(\mathbf{r})=(\mathcal{S} / L) \mathcal{M}(\mathbf{r}),
$$

with the "initial condition"

$$
Z\left(L^{\prime}=0, \mathbf{r}\right)=1
$$

and the boundary condition

$$
Z\left(L^{\prime}, \mathbf{r} \rightarrow S\right) \rightarrow 0
$$

on the particle surface $S$. The bulk-normalized polymer density profile of the ideal chains is given by ${ }^{6}$

$$
\mathcal{M}(\mathbf{r})=\frac{1}{L} e^{\mathcal{S}} \int_{0}^{L} d L^{\prime \prime} Z\left(L^{\prime \prime}, \mathbf{r}\right) Z\left(L-L^{\prime \prime}, \mathbf{r}\right) .
$$

Note that for $\mathbf{r}$ far from the particle or wall, $Z\left(L^{\prime}, \mathbf{r}\right)$ approaches the $\mathbf{r}$-independent value $e^{-b N N^{\prime} n}$, so that $\mathcal{M}(\mathbf{r})$ approaches 1 .

In the mean-field approximation the free energy cost $F$ of immersing the particle is also determined by $\mathcal{M}$ and is given by

$$
\frac{F}{k_{B} T}=V \frac{\Pi}{k_{B} T}+n \int d \mathbf{r}\left\{1-\mathcal{M}(\mathbf{r})+\frac{\mathcal{S}}{2}\left[1-\mathcal{M}^{2}(\mathbf{r})\right]\right\},
$$

as we show in Appendix B. Here $V$ is the volume occupied by the particle, and the integral extends over the volume outside the particle. Note that the $\mathcal{M}$-dependent terms in the integrand have the form of the bulk-pressure in Eqs. (2.2), (2.3), with the polymer density in the bulk $n$ replaced by the local density $n \mathcal{M}(\mathbf{r})$.

\section{B. Spherical and cylindrical particles}

The closed system of Eqs. (2.8)-(2.12) determining $\mathcal{M}(\mathbf{r})$ and Eq. (2.13) for $F$ apply to arbitrary particle shapes. For a sphere or an infinitely long cylinder, $\mathcal{M}(\mathbf{r})$ and $Z\left(L^{\prime}, \mathbf{r}\right)$ only depend on the distance $r$ from the center of the sphere or on the distance $r_{\perp}$ from the axis of the cylinder.

A useful concept is a "generalized cylinder" ${ }^{13,16}$ with an "axis" of $d_{\|}$dimensions and with the remaining $d-d_{\|}$ $=d_{\perp}$ dimensions perpendicular to the axis. The outer space of the generalized cylinder is determined by distances $r_{\perp}$ from the axis larger than its "radius" $R$. For $d_{\|}=0$ the generalized cylinder becomes a sphere. For $d_{\|}=1$ and $d=3$ it is a cylinder of radius $R$, and for $d_{\|}=2, d=3$ it is a plate of thickness $2 R$ in three dimensions. Below we shall consider a generalized cylinder in $d=4-\epsilon$ dimensions with

$$
d_{\|}=1-\epsilon, \quad d_{\perp}=3,
$$

which tends for $\epsilon \backslash 0$ to a cylinder in $d=4$ dimensions and for $\epsilon \nearrow 1$ to a sphere in $d=3$ dimensions. Another route leading to a sphere in three dimensions is given by $d_{\|}=0$, $d_{\perp}=4-\epsilon$. Although either of the two routes can be used to estimate excluded volume effects in $\mathcal{M}$ or $F$ for a sphere in $d=3$, we prefer the route in Eq. (2.14) because of the better starting value for the small radius ${ }^{33}$ exponent $d_{\perp}-(1 / \nu)$. In this section we determine the leading order results near $d$ $=4$, for which the tree- or mean-field approximation applies. Although the leading order results lead to estimates for scaling functions in $d=3$ with only moderate quantitative success, they demonstrate in a transparent way fundamental properties, such as the density-pressure identity Eq. (1.3) or the small radius expansion, ${ }^{13,16}$ which should apply along the whole route. For more accurate quantitative estimates for $d$ $=3$ see Sec. III below. 
According to Eqs. (2.8) -(2.12) in mean-field approximation the functions $Z\left(L^{\prime}, \mathbf{r}\right), \mathcal{V}(\mathbf{r})$, and $\mathcal{M}(\mathbf{r})$, for ${ }^{34}$ given $R$, $L$, and $\mathcal{S}$, only depend on $r_{\perp}$ and $d_{\perp}$ and are independent ${ }^{35}$ of $\mathbf{r}_{\|}$and $d_{\|}$. On using route Eq. (2.14), one finds that the scaling function $m$ of the density profile ${ }^{36}$

$$
\mathcal{M}(\mathbf{r})=m_{d}\left(\frac{r_{\perp}-R}{\mathcal{R}_{x}}, \frac{R}{\mathcal{R}_{x}}, \frac{n}{n^{*}}\right)
$$

to leading order in $d / 4$ equals the corresponding meanfield scaling function for a sphere in three dimensions. Its explicit form is obtained in Subsections E, F, G below.

In order to obtain a finite free energy of immersion $F$ we have to consider a generalized cylinder with an "axis" of large but finite extent or "volume"

$$
V_{\|}=v\left(\lambda / 2, d_{\|}\right)
$$

which is characterized by a large radius $\lambda / 2$. Here

$$
v(\lambda / 2, d) \equiv \Omega_{d} \int_{0}^{\lambda / 2} d r r^{d-1}=(\lambda / 2)^{d} \pi^{d / 2}[\Gamma(1+d / 2)]^{-1}
$$

is the volume of a sphere with radius $\lambda / 2$, and $\Omega_{d}$ is the surface area of a sphere with radius 1 , in $d$ dimensions. Note that

$$
V_{\|}=\lambda, 1 \quad \text { for } \quad d_{\|}=1,0 .
$$

In the mean-field approximation the free energy cost per unit "axis-volume" $V_{\|}$and per $k_{B} T, F /\left(k_{B} T V_{\|}\right)$, follows from the right hand side of Eq. (2.13) on replacing $V$ by the crosssectional volume $V_{\perp}=v\left(R, d_{\perp}\right)$ and the integration $\int d \mathbf{r}$ by an integration $\int d \mathbf{r}_{\perp}$ over that part of the "plane" $r_{\|}=0$ perpendicular to the axis which is outside the particle:

$$
F \rightarrow F / V_{\|}: \quad V \rightarrow V_{\perp} ; \quad \int d \mathbf{r} \rightarrow \int d \mathbf{r}_{\perp}, \quad r_{\perp}>R .
$$

Since end effects can be neglected for $R, \mathcal{R}_{x} \ll \lambda$, and since the mean-field profile $\mathcal{M}(\mathbf{r})$ is independent of $\mathbf{r}_{\|}, d_{\|}$, the free energy cost per unit axis volume in the mean-field approximation Eqs. (2.13), (2.19) only depends on $d_{\perp}$ and is independent of $d_{\|} \cdot{ }^{13,16}$ For the case of route Eq. (2.14), in which for dimensional reasons ${ }^{36}$

$$
\frac{F}{k_{B} T V_{\|}}=n \mathcal{R}_{x}^{3} f_{d}\left(\frac{R}{\mathcal{R}_{x}}, \frac{n}{n^{*}}\right)
$$

the scaling function $f_{d}$ in leading order $d / 4$ equals the corresponding mean-field scaling function for a sphere in three dimensions.

Figure 2 shows numerical results for the scaling function $f_{4}$ versus the size ratio $\rho=R / \mathcal{R}_{x}$, for various values of the overlap variable $\mathcal{S}=n / n^{*}$. The numerical procedure for solving Eqs. (2.8)-(2.12) is described in Appendix C. Note the $\mathcal{S}$-independent behavior $f_{4} \rightarrow 2 \pi \rho$ for small size ratio $\rho$ which is in agreement with Eq. (1.5). For large size ratio, $f_{4}=(4 \pi / 3) \rho^{3}(1+\mathcal{S} / 2)$, in agreement with Eq. (2.28) below.

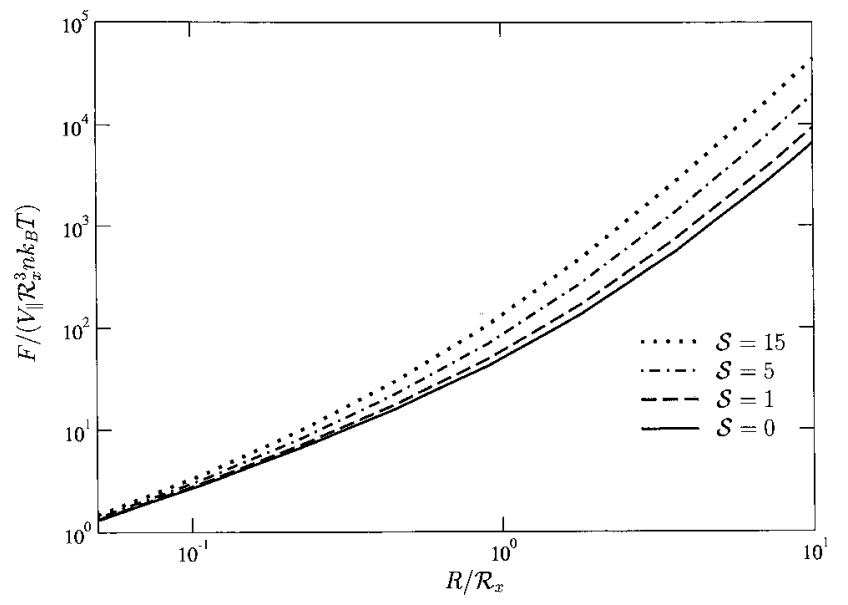

FIG. 2. Scaled solvation free energy $F /\left(V_{\|} \mathcal{R}_{x}^{3} n k_{B} T\right)$ vs size ratio $\rho$ $=R / \mathcal{R}_{x}$ for various values of the inter-chain overlap $\mathcal{S}=n / n^{*}$. Shown is the scaling function $f_{4}$ [Eq. (2.20)] for a cylinder of infinite length $V_{\|}=\lambda \rightarrow \infty$ in $d=4$ dimensions. This also furnishes a qualitative estimate of the corresponding scaling function $f_{3}$ for a sphere $\left(V_{\|}=1\right)$ in $d=3$ dimensions. Note the crossover from the overlap-independent result $2 \pi \rho$ for small $\rho$ [Eq. (1.5)] to the result $(4 \pi / 3) \rho^{3}(1+\mathcal{S} / 2)$ for large $\rho$ [Eq. (2.28)]

\section{Density-pressure identity}

The pressure exerted by the polymers onto a given surface element of an embedded mesoscopic particle is proportional to the local polymer density $n \mathcal{M}^{\text {(as) }}(\mathbf{r})$ near the surface element. ${ }^{19,20}$ With the density taken from the scaling regime ${ }^{21}$ the factor of proportionality involves a universal constant $B$, which can be calculated and leads to the identity Eq. (1.3). In Appendix A we prove the identity for the case $d \nearrow 4$, i.e., within the mean-field or tree approximation, in which

$$
1 / \nu=2, \quad B=2 .
$$

For the generalized cylinder the pressure $p$ acts on a surface $S$ with magnitude $S_{\perp} V_{\|}$and with the surface-normal perpendicular to the axis. Thus the identity Eq. (1.3) reads

$$
\mathcal{M}\left(r_{\perp} \searrow R\right)=\mathcal{M}^{\text {as }}\left(r_{\perp}\right)=2\left(\frac{r_{\perp}-R}{\mathcal{R}_{x}}\right)^{2} \frac{p}{n k_{B} T},
$$

with

$$
p=\frac{1}{S_{\perp}} \frac{d}{d R} \frac{F}{V_{\|}} .
$$

Here $S_{\perp}=R^{d_{\perp}-1} \Omega_{d_{\perp}}$ and $\Omega_{d}=2 \pi^{d / 2} / \Gamma(d / 2)$. In particular,

$$
S_{\perp}=4 \pi R^{2}, \quad d_{\perp}=3
$$

for the case of a cylinder of type of Eq. (2.14) in which $d_{\perp}$ $=3$, and $S_{\perp}=2$ for the case of a plate in which $d_{\perp}=1$.

Figure 3 shows numerical results for $p /\left(n k_{B} T\right)$ as a function of the size ratio $R / \mathcal{R}_{x}$ for various values of the overlap $\mathcal{S}$.

\section{Number of missing chains}

A simple way to quantify the polymer depletion effect is by considering the decrease $\langle\delta \mathcal{N}\rangle$ of the number $\langle\mathcal{N}\rangle$ of chains in the monodisperse polymer solution on inserting the 


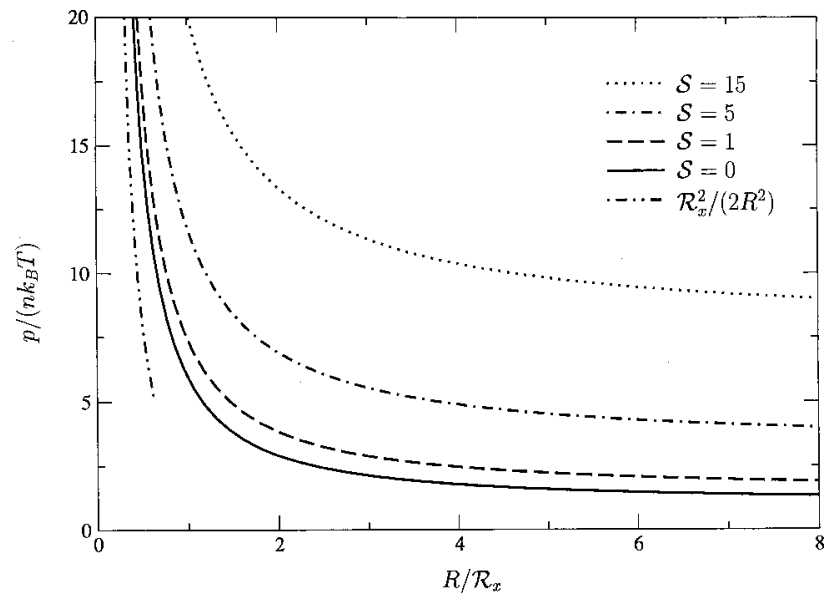

FIG. 3. Scaled polymer-pressure $p /\left(n k_{B} T\right)$ on the surface of a cylindrical rod in $d=4$ dimensions as a function of the size ratio $\rho=R / \mathcal{R}_{x}$ for various values of the overlap $\mathcal{S}$. This result also furnishes a qualitative estimate of the pressure on the surface of a spherical particle in $d=3$ dimensions. Note the crossover from the $\mathcal{S}$-independent behavior $1 /\left(2 \rho^{2}\right)$ for small $\rho$ [Eqs. (1.5), (2.23), (2.24)] to the $\rho$-independent behavior $1+\mathcal{S} / 2$ for large $\rho$ [Eq. (2.29)].

nonadsorbing particle. In a grand canonical ensemble with chain fugacity $\zeta$, the number decrease per unit axis "volume" is given by

$$
\begin{aligned}
\langle-\delta \mathcal{N}\rangle / V_{\|} & =\zeta \frac{d}{d \zeta} \sum_{\mathcal{N}=1}^{\infty} \frac{\zeta^{\mathcal{N}}}{\mathcal{N} !}\left(\left[\mathcal{Z}_{c}^{(\mathcal{N})}\right]_{\text {no particle }}-\mathcal{Z}_{c}^{(\mathcal{N})}\right) / V_{\|} \\
& =\zeta \frac{d}{d \zeta} \frac{F / V_{\|}}{k_{B} T} \\
& =n\left(V_{\perp}+\int_{r_{\perp}>R} d \mathbf{r}_{\perp}\left[1-\mathcal{M}\left(r_{\perp}\right)\right]\right)
\end{aligned}
$$

Here $\mathcal{Z}_{c}^{(\mathcal{N})}$ is the connected part of the partition function of

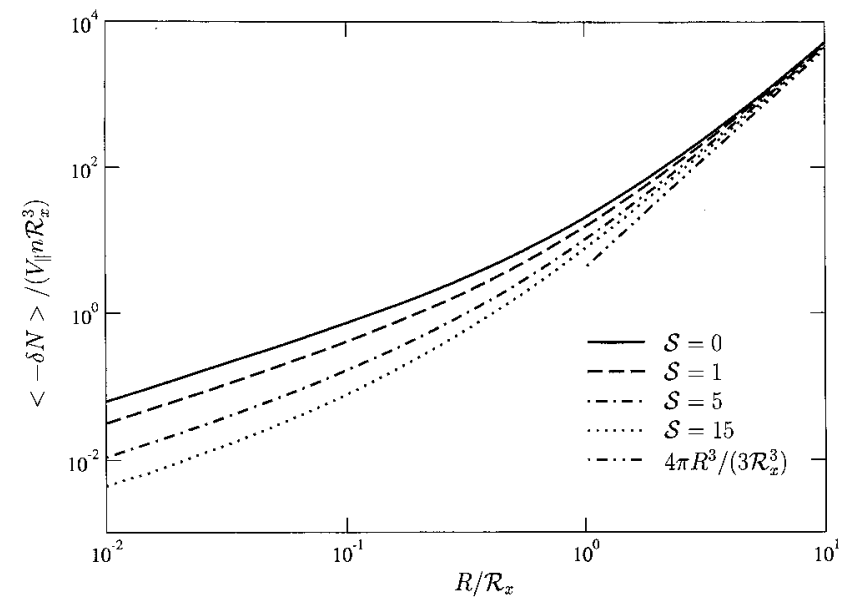

FIG. 4. Scaled number of missing chains $\langle-\delta \mathcal{N}\rangle /\left(V_{\|} n \mathcal{R}_{x}^{3}\right)$ vs size ratio $\rho=R / \mathcal{R}_{x}$ for various values of the interchain overlap $\mathcal{S}$ [compare Eq. (2.25)]. The result shown is for a cylinder in $d=4$ dimensions with axis length $V_{\|}=\lambda$. It also gives a qualitative estimate for the case of a spherical particle in $d=3$ in which $V_{\|}=1$. Note the crossover from the result $2 \pi \rho /(1+\mathcal{S})$ for small $\rho$ [Eq. $(2.44)]$ to the overlap-independent behavior $(4 \pi / 3) \rho^{3}$ for large $\rho$ [Eq. (2.38)].
$\mathcal{N}$ chains. The relationship between $\zeta$ and $n$ in the mean-field approximation is given in Eqs. (B4) and (B6) of Appendix B.

Figure 4 shows the number $\langle-\delta \mathcal{N}\rangle$ of missing chains as a function of the size ratio $\rho=R / \mathcal{R}_{x}$ for various values of the overlap variable $\mathcal{S}$. Analytic expressions for $\langle-\delta \mathcal{N}\rangle$ in the special cases $R \gg \mathcal{R}_{x}$ and $R \ll \mathcal{R}_{x}$ will be given in subsections $\mathrm{E}$ and $\mathrm{F}$ below.

\section{E. Planar wall and large particle}

Consider a large particle with a smooth surface and with all local principal radii $R_{i}$ of surface-curvature much larger than the polymer lengths. Then one expects ${ }^{16,29}$ a smallcurvature expansion of the Helfrich type

$$
F=V \Pi+\int d S\left[\sigma+\kappa K_{m}+\ldots\right]
$$

for the free energy of immersion $F$. Here

$$
K_{m}=\frac{1}{2} \sum_{i=1}^{d-1} \frac{1}{R_{i}}
$$

is proportional to the local mean surface-curvature, and the coefficients $\sigma$ and $\kappa$, which denote the surface tension and the coefficient of spontaneous curvature, respectively, are independent of the shape of the weakly curved surface.

For the generalized cylinder Eq. (2.26) reduces to

$$
\frac{F}{V_{\|}}=V_{\perp} \Pi+S_{\perp}\left[\sigma+\frac{d_{\perp}-1}{2} \frac{\kappa}{R}+\ldots\right],
$$

and Eq. (2.23) leads to a pressure

$$
p=\Pi+\left(d_{\perp}-1\right) \frac{\sigma}{R}+\frac{\left(d_{\perp}-1\right)\left(d_{\perp}-2\right)}{2} \frac{\kappa}{R^{2}}+\ldots
$$

on the surface with surface normal perpendicular to the axis. Here we have used relations such as $d V_{\perp} / d R=S_{\perp}$. The form

$$
\sigma=n k_{B} T \int_{0}^{\infty} d z\left(1-\mathcal{M}_{\mathrm{pw}}(z)+\frac{\mathcal{S}}{2}\left[1-\mathcal{M}_{\mathrm{pw}}^{2}(z)\right]\right)
$$

of the surface tension $\sigma$ follows from a comparison of Eqs. (2.13) and (2.26) for the case of a half space with a planar wall surface in which all the $1 / R_{i}$ vanish. ${ }^{37}$ Here $z$ denotes the distance of point $\mathbf{r}$ from the planar wall. Since $\sigma$ is positive, we conclude from Eq. (2.29) that a weakly curved convex surface (such as the surface of a spherical or cylindrical particle with large but finite radius $R$ ) experiences a larger pressure $p$ from the polymer chains than a planar wall (with $R=\infty)$. This is in accordance with the smaller entropy loss of polymer chains near a convex surface. See also Fig. 3 and Eq. (2.37) below.

The scaling form of the surface tension,

$$
\frac{\sigma}{k_{B} T}=n \mathcal{R}_{x} g(\mathcal{S})
$$

follows from Eqs. (2.30) and (2.15). In the dilute ${ }^{17}$ and semidilute limit one finds ${ }^{38}$ the analytic expressions 


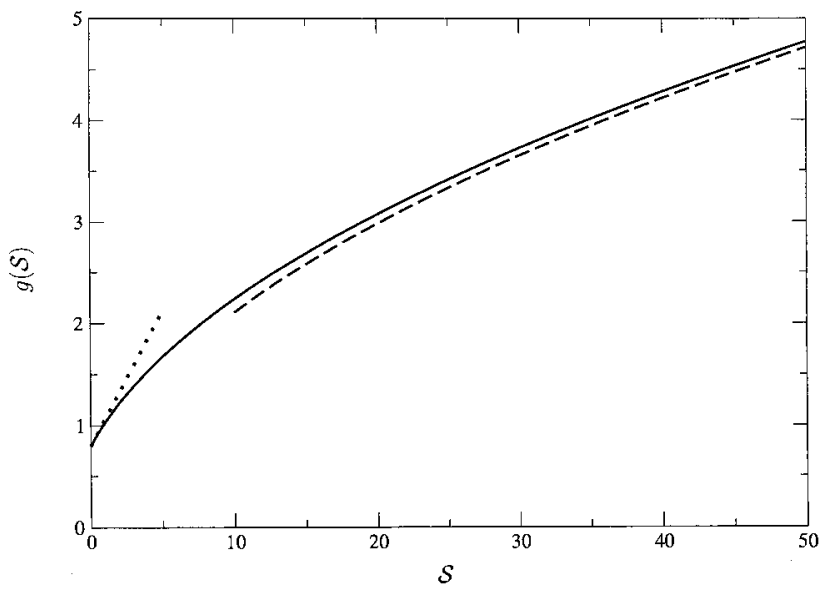

FIG. 5. Scaling function $g(\mathcal{S})$ of the surface tension $\sigma=k_{B} T n \mathcal{R}_{x} g$ in the mean-field approximation [Eq. (2.31)]. The dotted and dashed lines show the asymptotic behavior for small and large $\mathcal{S}$, respectively, see Eq. (2.32).

$$
\begin{aligned}
g(\mathcal{S}) \rightarrow & \left(\sqrt{\frac{2}{\pi}}\left[1+\mathcal{S} \lambda_{1}+\mathcal{O}\left(\mathcal{S}^{2}\right)\right], \quad \frac{2}{3} \mathcal{S}^{1 / 2}\right), \\
\mathcal{S} \rightarrow(0, \infty) &
\end{aligned}
$$

with

$$
\lambda_{1}=\frac{5 \sqrt{5}}{3}-\frac{12 \sqrt{2}}{5}=0.333 .
$$

Figure 5 shows the scaling function $g(\mathcal{S})$ for arbitrary $\mathcal{S}$, which we have obtained numerically from Eqs. (2.8)-(2.12) and (2.30).

For the coefficient of spontaneous curvature one finds ${ }^{38}$

$$
\frac{\kappa}{k_{B} T}=n \mathcal{R}_{x}^{2} h(\mathcal{S}),
$$

with

$$
h(\mathcal{S}) \rightarrow\left(\frac{1}{2}+\mathcal{S} \lambda_{2}, \quad \frac{1}{3}[4 \ln 2-1]\right), \quad \mathcal{S} \rightarrow(0, \infty),
$$

where

$$
\lambda_{2}=\frac{1}{8}\left[19-\frac{1}{\pi}\left(17+\frac{75}{2} \arctan 2\right)\right]=0.047 .
$$

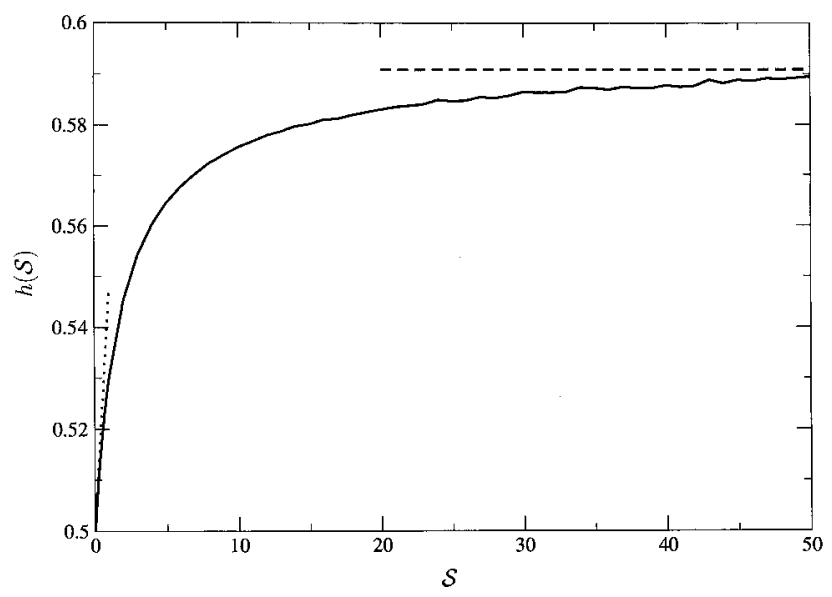

FIG. 6. Scaling function $h(\mathcal{S})$ of the coefficient $\kappa=k_{B} \operatorname{Tn} \mathcal{R}_{x}^{2} h$ of the spontaneous curvature in the mean-field approximation [Eqs. (2.28) and (2.34)]. The dotted and dashed lines show the asymptotic behavior for small and large $\mathcal{S}$, respectively, see Eq. (2.35).

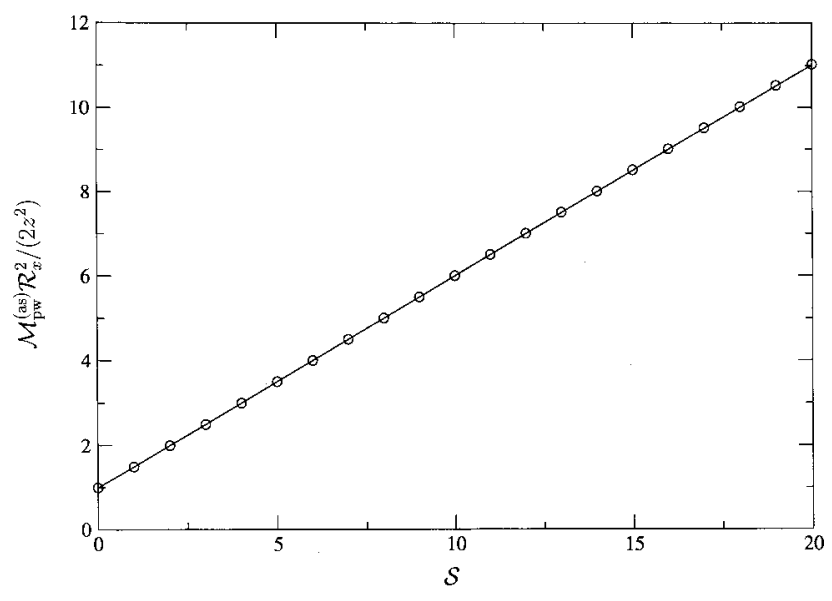

FIG. 7. Density-pressure identity for a planar wall [see Eq. (2.37) with $r_{\perp}$ $-R=z$ finite and $R=\infty]$. The amplitude $\mathcal{M}^{\text {as }} \mathcal{R}_{x}^{2} /\left(2 z^{2}\right)$ of the density profile $\mathcal{M}=\mathcal{M}_{\mathrm{pw}}$ (circles) reproduces the scaled osmotic pressure $\Pi /\left(n k_{B} T\right)$ $=1+\mathcal{S} / 2$ (full line) very well.

Figure 6 shows the scaling function $h(\mathcal{S})$ for arbitrary $\mathcal{S}$, which we have obtained numerically.

Now let us turn to the normalized monomer density profile $\mathcal{M}$ near a planar wall or near a generalized cylinder of large radius $R$. For $\mathbf{r}$ close to the surface the density pressure identity Eq. (2.22) predicts

$$
\begin{aligned}
\mathcal{M}\left(r_{\perp} \searrow R\right)= & \mathcal{M}^{(\text {as })}\left(r_{\perp}\right) \\
= & 2\left(\frac{r_{\perp}-R}{\mathcal{R}_{x}}\right)^{2}\left[1+\frac{\mathcal{S}}{2}+\left(d_{\perp}-1\right) \frac{\mathcal{R}_{x}}{R} g(\mathcal{S})\right. \\
& \left.+\frac{\left(d_{\perp}-1\right)\left(d_{\perp}-2\right)}{2}\left(\frac{\mathcal{R}_{x}}{R}\right)^{2} h(\mathcal{S})+\ldots\right] .
\end{aligned}
$$

Here we have inserted the pressure $p$ given by Eq. (2.29) and by Eqs. (2.2), (2.31), and (2.34) into the identity Eq. (2.22). Figures 7 and 8 show that the numerically determined profile

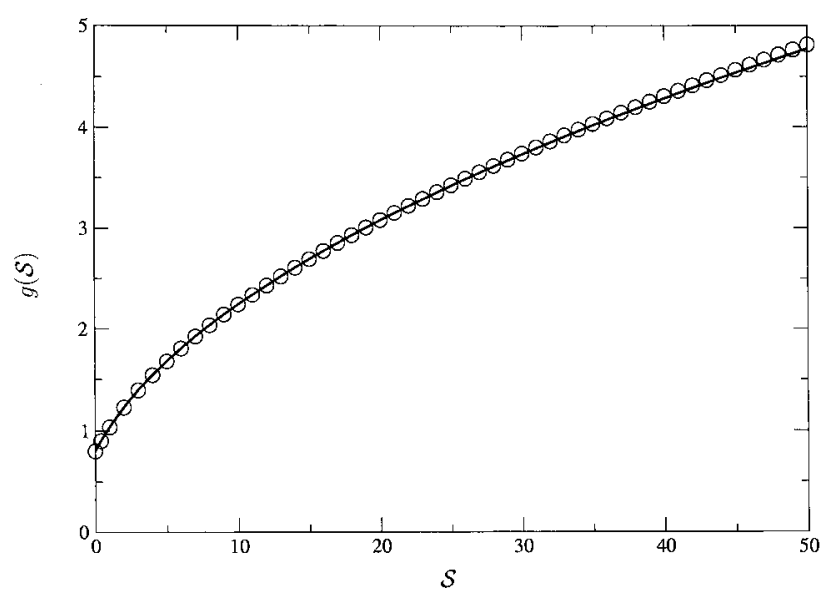

FIG. 8. Contribution of the surface tension to the density-pressure identity for a weakly curved surface of a cylinder [see Eq. (2.37) with $d_{\perp}=3$ ]. The limit $\lim _{R / \mathcal{R}_{x} \rightarrow \infty}\left(R /\left(2 \mathcal{R}_{x}\right)\right)\left[\left[\mathcal{M}^{\text {(as) }} \mathcal{R}_{x}^{2} /\left(2\left(r_{\perp}-R\right)^{2}\right)\right]-(1+(\mathcal{S} / 2))\right]$ taken from the density profile near the surface $\mathcal{M}^{\text {(as) }}$ is well approximated by the value for $R / \mathcal{R}_{x}=100$ (circles) and reproduces the scaling function $g(\mathcal{S})$ of the surface tension (full line). 
$\mathcal{M}$ for a cylinder of type Eq. (2.14) with $d_{\perp}=3, d \nearrow 4$ and a large radius $R$ does indeed fulfill the density-pressure identity Eq. (2.22).

Besides this numerical check, we have also checked Eq. (2.22) analytically. In Appendix E we show that in the semidilute limit $\mathcal{S} \rightarrow \infty$ the expressions $\mathcal{S} / 2$ and $\frac{2}{3} \mathcal{S}^{1 / 2}$ of, respectively, $\Pi /\left(n k_{B} T\right)$ and $g$ do indeed appear in the asymptotic profile $\mathcal{M}^{\text {(as) }}$, in accordance with Eq. (2.37).

In the dilute limit $\mathcal{S} \rightarrow 0$ and the semidilute limit $\mathcal{S} \rightarrow \infty$ the normalized density profiles $\mathcal{M}_{\mathrm{pw}}$ near a planar wall are known in analytic form. ${ }^{9,19,39}$ In particular, for $\mathcal{S} \rightarrow \infty, \mathcal{M}_{\mathrm{pw}}$ $=\operatorname{Tanh}^{2}\left(\mathcal{S}^{1 / 2} z / \mathcal{R}_{x}\right)$, and the number of missing chains near a large particle per unit surface area is given by

$$
\begin{aligned}
& {[\langle-}\left.\delta \mathcal{N}\rangle / V_{\|}-n \frac{4 \pi}{3} R^{3}\right] /\left(4 \pi R^{2}\right) \\
& \quad=n \mathcal{R}_{x}\left[(2 / \pi)^{1 / 2}, \mathcal{S}^{-1 / 2}\right], \quad \mathcal{S} \rightarrow[0, \infty] .
\end{aligned}
$$

Note that the distance $\xi_{I}^{(\mathrm{pw})}$ from the planar wall of the point of inflection of $\mathcal{M}_{\mathrm{pw}}$ is also proportional to $\mathcal{R}_{x}$ and $\mathcal{R}_{x} / \mathcal{S}^{1 / 2}$ for $\mathcal{S} \rightarrow 0$ and $\mathcal{S} \rightarrow \infty$, respectively.

\section{F. Thin cylinder and small sphere}

Here we consider the case $R \ll \mathcal{R}_{x}, \xi$, in which the radius $R$ of the cylinder Eq. (2.14) in $d / 4$ is much smaller than the characteristic mesoscopic polymer lengths. The result Eq. (1.5) for the solvation free energy of a thin cylinder in $d$ $=4$ is based on a reduction factor

$$
W_{\text {cy }}\left[\mathbf{r}_{P, j}\right] \rightarrow 1-A_{\text {id }}\left(d_{\perp}\right) R^{d_{\perp}-2} \mathcal{R}_{x}^{2} \int d \mathbf{r}_{\|} \Theta\left(\mathbf{r}_{\perp}=0, \mathbf{r}_{\|}\right)
$$

of the Boltzmann weight for chain configurations. Here

$$
\Theta\left(\mathbf{r}_{\perp}, \mathbf{r}_{\|}\right)=\sum_{P=1}^{\mathcal{N}} \frac{1}{N} \sum_{j=1}^{N} \delta\left(\mathbf{r}_{\perp ; P, j}-\mathbf{r}_{\perp}\right) \delta\left(\mathbf{r}_{\| ; P, j}-\mathbf{r}_{\|}\right)
$$

is the configuration dependent density of chains at the point $\mathbf{r}=\left(\mathbf{r}_{\perp}, \mathbf{r}_{\|}\right)$, the vector $\mathbf{r}_{P, j}$ is the position of monomer $j$ in chain $P$, and $A_{\text {id }}\left(d_{\perp}=3\right)$ equals $^{13,16,23} 2 \pi$. Equations (2.39), (2.40) can also be used to evaluate the normalized density profile $\mathcal{M}\left(r_{\perp}\right)$ for the case $R \ll r_{\perp}, \mathcal{R}_{x}, \xi$. This is given by

$$
\begin{aligned}
\mathcal{M}\left(r_{\perp}\right)= & \left\langle\Theta\left(\mathbf{r}_{\perp}, \mathbf{r}_{\|}\right)\right\rangle_{\mathrm{cyl}} / n \rightarrow 1-2 \pi R \mathcal{R}_{x}^{2} \\
& \times \int d \mathbf{r}^{\prime}{ }_{\|}\left\langle\Theta\left(\mathbf{r}_{\perp}, \mathbf{r}_{\|}\right) \Theta\left(0, \mathbf{r}_{\|}^{\prime}\right)\right\rangle_{c, \text { bulk }} / n \\
= & 1-2 \pi R \mathcal{R}_{x}^{2} \int \frac{d^{3} p}{(2 \pi)^{3}} e^{i \mathbf{p r}_{\perp}} \frac{D\left(p^{2} L\right)}{1+\mathcal{S} D\left(p^{2} L\right)},
\end{aligned}
$$

where $D$ is the Debye function

$$
D(x)=2\left(\mathrm{e}^{-x}-1+x\right) / x^{2} .
$$

In the last step in Eq. (2.41) we inserted the tree expression of the bulk density correlation function ${ }^{8}$ and used $d_{\perp}=3$. In the limit $R \ll r_{\perp} \ll \mathcal{R}_{x}, \xi$, the above expression reduces to

$$
\begin{aligned}
\mathcal{M}\left(r_{\perp}\right) & \rightarrow 1-2 \pi R \mathcal{R}_{x}^{2} \int \frac{d^{3} p}{(2 \pi)^{3}} e^{i \mathbf{p} \mathbf{r}_{\perp}} \frac{2}{p^{2} L} \\
& =1-2 R / r_{\perp} .
\end{aligned}
$$

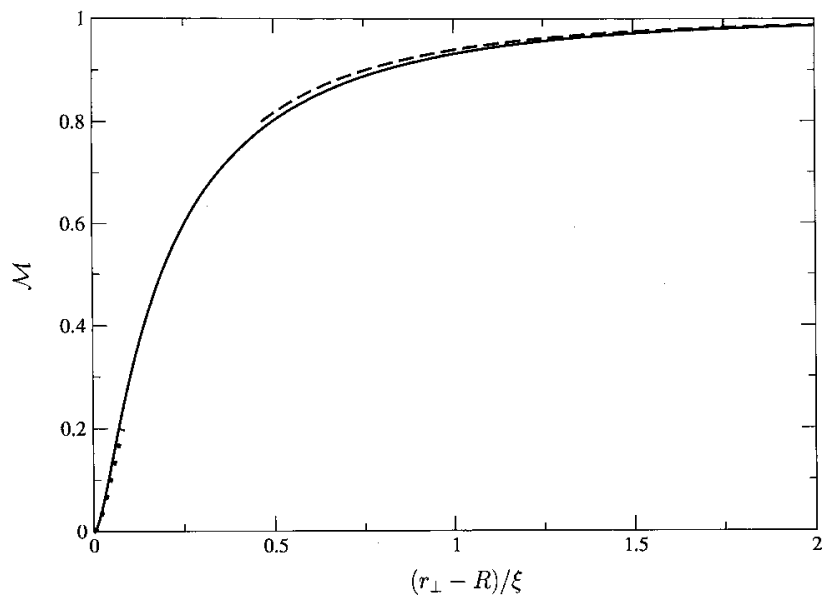

FIG. 9. The mean-field density profile around a thin cylinder in a semidilute solution near four dimensions provides a qualitative estimate for the density profile around a small sphere in a semidilute solution in three dimensions. The full curve shows numerical data for the length ratios $R: \xi: \mathcal{R}_{x}$ $=1: 10: 100$ which interpolate smoothly between the limiting behaviors Eqs. (1.4) and (E16) which are also shown.

Equation (2.43) is consistent with the right hand side of Eq. (1.4), which describes the behavior of $\mathcal{M}\left(r_{\perp}\right)$ close to the surface of the cylinder Eq. (2.14), i.e., for $R, r_{\perp} \ll \mathcal{R}_{x}, \xi$. The profile in the semidilute limit for $R \ll r_{\perp}, \xi \ll \mathcal{R}_{x}$ is given in Eqs. (E16) and (E17) of Appendix E. Figure 9 shows our numerical result for the density profile of a thin cylinder in semidilute solution which smoothly interpolates between the limits of Eqs. (1.4) and (E16).

The expressions for the cylinder Eq. (2.14) in Eqs. (1.4) and (1.5) obey the density-pressure relations Eqs. (2.22) and (2.23). Inserting $F /\left(k_{B} T \lambda\right)=2 \pi n R \mathcal{R}_{x}^{2}$ into Eq. (2.23) with $V_{\|}=\lambda$ and with $S_{\perp}$ from Eq. (2.24) leads in Eq. (2.22) to an expression $\mathcal{M}^{\text {(as) }}\left(r_{\perp}\right)=\left(r_{\perp}-R\right)^{2} / R^{2}$, which is consistent with the expression Eq. (1.4). Apart from the trivial linear $n$-dependence of $F$ these expressions are independent of the inter-chain overlap. This contrasts with the number of missing chains due to the presence of a thin cylinder or a small sphere,

$$
\langle-\delta \mathcal{N}\rangle / V_{\|}=2 \pi n R \mathcal{R}_{x}^{2} /(1+\mathcal{S}),
$$

which follows from Eqs. (2.25) and (2.41) and has a nontrivial dependence on the overlap $\mathcal{S}$.

\section{G. Form of the density profiles}

Figure 10 shows our numerical results for the density profiles $\mathcal{M}_{\text {pw }}$ of a planar wall versus the scaled distance $\left(r_{\perp}-R\right) / \mathcal{R}_{x}=z / \mathcal{R}_{x}$ for various values of the overlap $\mathcal{S}$. Figure 11 shows the distance $\xi_{I}^{(\mathrm{pw})}=z_{I}$ of the point of inflection from the wall as a function of $\mathcal{S}$. This should be compared with $^{31}$ the density correlation length $\xi_{D}$ of the bulk solution which is also indicated in Fig. 11. 


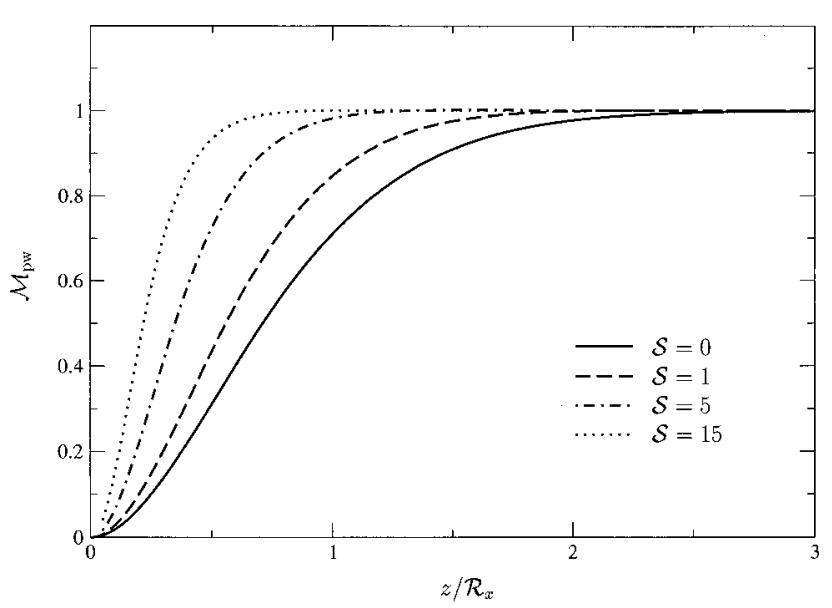

FIG. 10. Bulk-normalized density profiles $\mathcal{M}_{\mathrm{pw}}$ for a planar wall vs the scaled distance $z / \mathcal{R}_{x}$ from the wall for various values of the inter-chain overlap $\mathcal{S}$.

Using a lattice-walk model ${ }^{32}$ for polymers at the $\theta$ point, van der Gucht et al. ${ }^{40}$ found that the density profiles $\mathcal{M}_{\mathrm{pw}}$ approach their bulk value 1 in a nonmonotonic oscillatory way. Similar behavior was found in a recent Monte Carlo simulation of self avoiding lattice walks by Bolhuis et al. ${ }^{41}$ Our continuum mean-field theory for polymers in a good solvent near the upper critical dimension predicts a similar nonmonotonic behavior as shown in Fig. 12. The most pronounced maximum, which arises for an overlap $\mathcal{S} \approx 5$, is nearly twice as large as the largest maximum shown in Ref. 40 and less than a third of the largest maximum in Ref. 41. The scaled distance $z_{\max } / \mathcal{R}_{x}$ of the first maximum from the wall decreases with increasing overlap, as shown in Fig. 13. However, the decrease is much slower than that of either $\xi_{I}^{\mathrm{pw}} / \mathcal{R}_{x}$ or $\xi_{D} / \mathcal{R}_{x}$. This is also in qualitative agreement with the behavior reported in Refs. 40, 41.

Now we turn to the density profiles near a cylinder of finite radius. Figure 14 shows the distance $\xi_{I}=\left(r_{\perp}\right)_{I}-R$ of the point of inflection from the surface of the cylinder versus

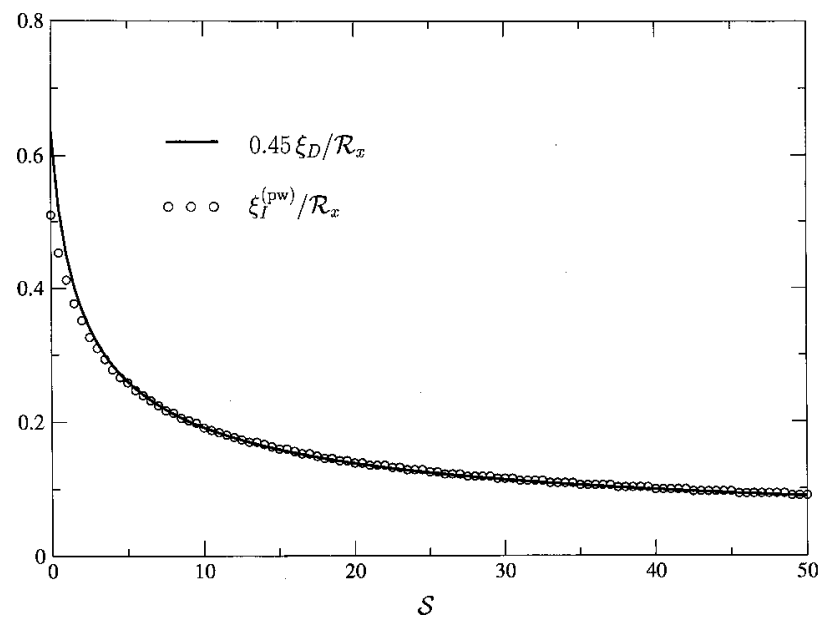

FIG. 11. Scaled distance $\xi_{I}^{(\mathrm{pw})} / \mathcal{R}_{x}$ from the planar wall of the point of inflection of the density profile vs the inter-chain overlap $\mathcal{S}$ (circles). The overlap-dependence of the bulk density correlation length (Ref. 31) $\xi_{D}$ with an adjusted prefactor is shown for comparison (full line).

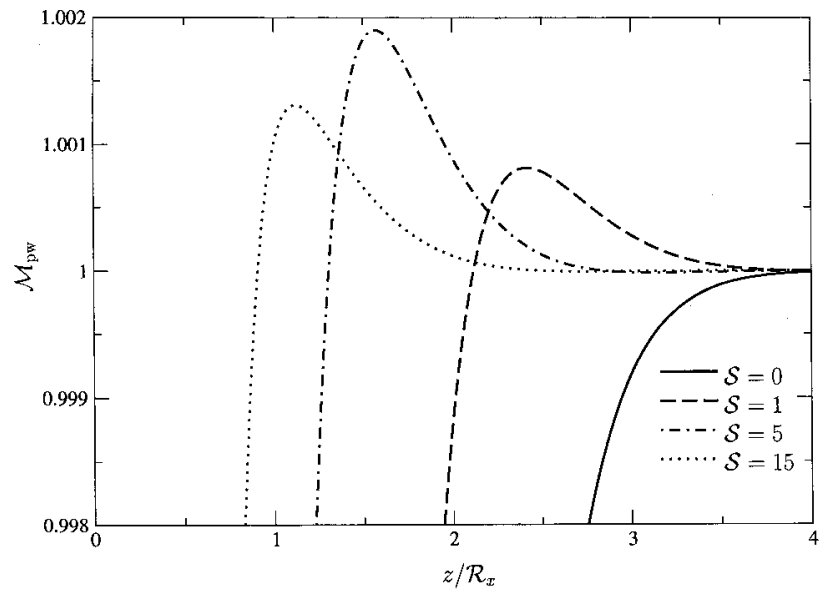

FIG. 12. Nonmonotonic behavior of the density profiles near a planar wall. Note the enlarged scale of the vertical axis. The most pronounced maximum occurs for an overlap of $\mathcal{S} \approx 5$ and is about twice as large as the largest maximum shown in Ref. 40 and less than a third of the largest maximum in Ref. 41.

the size ratio $\rho=R / \mathcal{R}_{x}$ for various values of the overlap $\mathcal{S}$. For $R \ll \mathcal{R}_{x}$ all the curves merge to approach the overlapindependent value $\xi_{I} \rightarrow R / 2$, corresponding to the point of inflection in Eq. (1.4).

The maximum of $\mathcal{M}$ found in the planar wall case persists for finite $\rho=R / \mathcal{R}_{x}$, although its height decreases with decreasing $\rho$, as seen in Fig. 15. Note that the highest maximum for given $\rho$ is always at $\mathcal{S} \approx 5$, independent of the value of $\rho$. Note also that the distance of the maximum from the surface divided by $\mathcal{R}_{x}$ only depends on $\mathcal{S}$ and is nearly independent of the size ratio $\rho$, i.e., the plot of Fig. 13 applies not only for $\rho=\infty$ but also for arbitrary $\rho$. The maximum persists all the way down to small size ratio $\rho \ll 1$, see the circles in Fig. 16, and it is correctly reproduced by ${ }^{42}$ the small radius expansion Eq. (2.41), which is shown as the full line in Fig. 16.

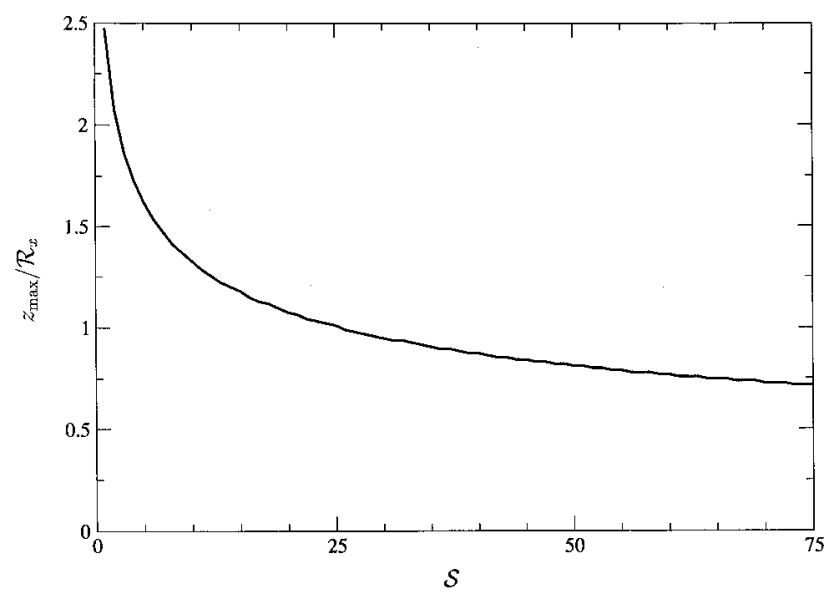

FIG. 13. Scaled distance $z_{\max } / \mathcal{R}_{x}$ of the maximum of the density profile from the planar wall vs the inter-chain overlap $\mathcal{S}$. Note that this distance decreases much more slowly with increasing overlap than $\xi_{I}^{(\mathrm{pw})} / \mathcal{R}_{x}$ and $\xi_{D} / \mathcal{R}_{x}$ in Fig. 11. 


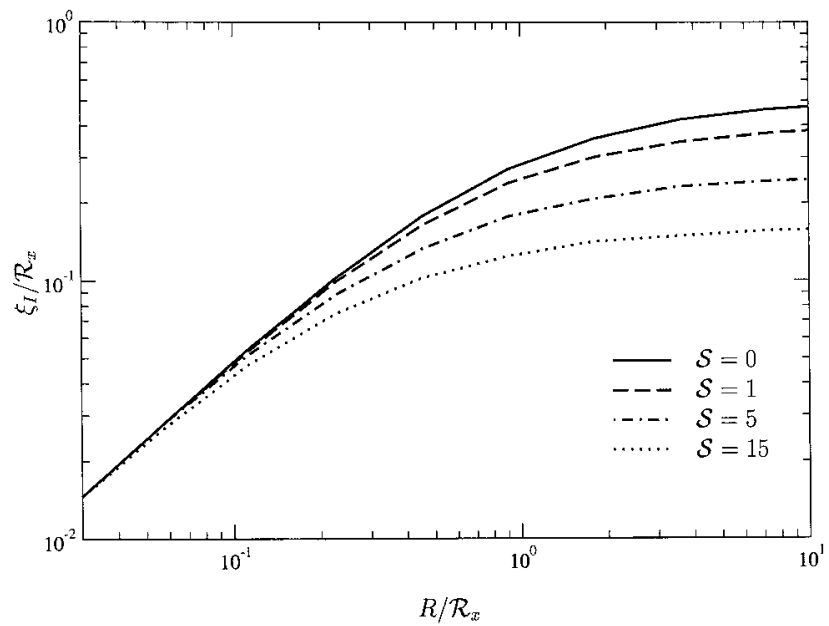

FIG. 14. Scaled distance $\xi_{I} / \mathcal{R}_{x}$ of the point of inflection of the density profile from the surface of a cylinder vs the size ratio $\rho=R / \mathcal{R}_{x}$, for various values of the inter-chain overlap $\mathcal{S}$. For small $\rho$ the scaled distance approaches the overlap-independent behavior $\rho / 2$ corresponding to the point of inflection in Eq. (1.4).

\section{RENORMALIZED TREE APPROXIMATION IN THREE DIMENSIONS}

The mean-field discussion in Sec. II is a first step toward obtaining the qualitative dependencies of various scaling functions on the size ratio and the inter-chain overlap. We now show that semi-quantitative results may be obtained with the renormalization group. On mapping the "critical" polymer system of interest with its long chains and large mesh size onto a "noncritical" system where mean-field theory applies approximately, we obtain scaling functions with the correct power-law exponents in three dimensions. These differ from the mean-field expressions.

Following the well known path of field theoretic renormalization, ${ }^{7-9}$ one reparameterizes ${ }^{43}$ the three basic variables $b, N, n$ in terms of "renormalized" variables $u_{r}, L_{r}, n_{r}$, where

$$
b / l^{4}=(4 \pi)^{d / 2} \mu^{\epsilon} Z_{u} u_{r} / 3,
$$

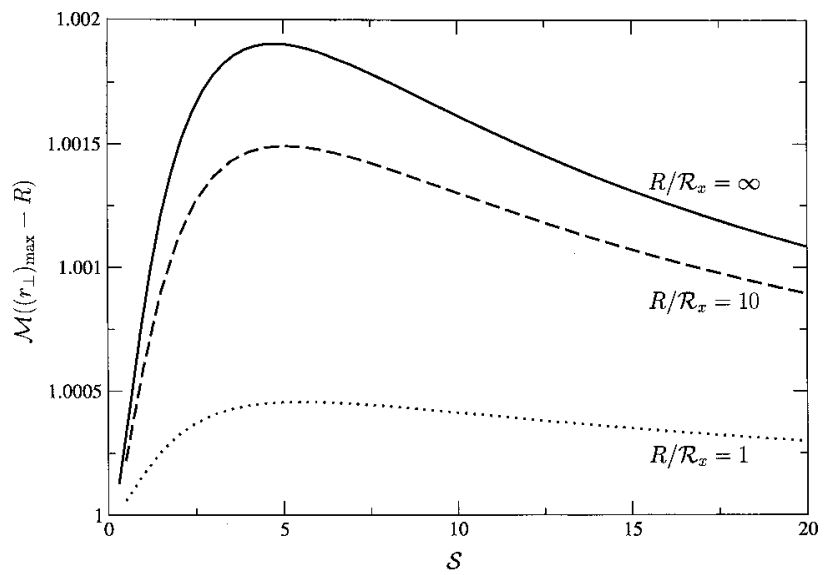

FIG. 15. Height of the maximum in the density profile near a cylindrical rod vs the inter-chain overlap $\mathcal{S}$, for various values of the size ratio $\rho=R / \mathcal{R}_{x}$. The highest maximum for given $\rho$ is always at $\mathcal{S} \approx 5$, independent of the value of $\rho$.

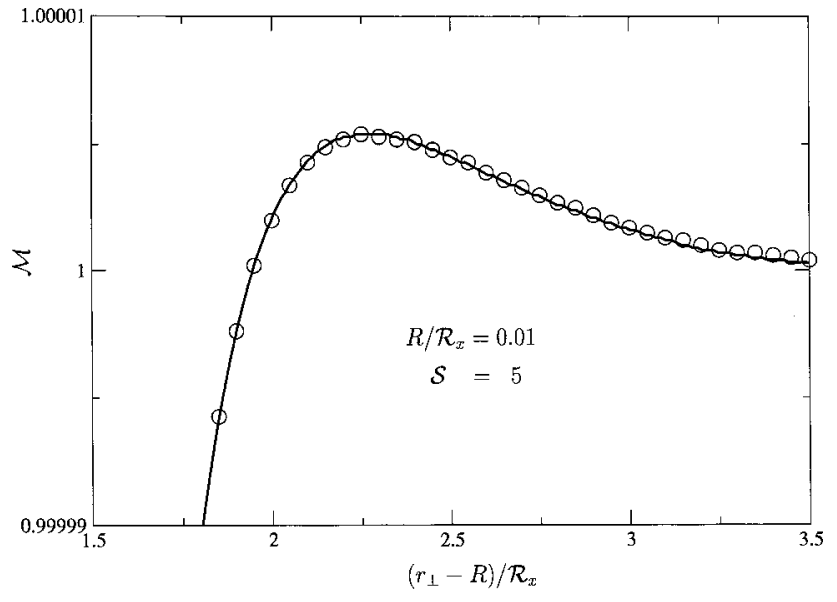

FIG. 16. Density profile with a maximum for a cylinder with $R \ll \mathcal{R}_{x}$ (circles). The maximum is well reproduced (full line) by the minimum in the bulk density correlation function on using the small radius expansion [see Eqs. (2.41) and (2.42)].

$$
\begin{aligned}
& N l^{2}=\mu^{-2} L_{r} / Z_{t}, \\
& n=\mu^{d} n_{r},
\end{aligned}
$$

and $Z_{u}, Z_{t}$ are renormalization factors. Since the inverse length scale parameter $\mu$ is arbitrary, one obtains a oneparameter family of renormalized theories. On changing the inverse length scale $\mu \rightarrow e^{-\lambda} \mu$, a critical polymer system with $L_{r}$ large and $n_{r} L_{r}$ small is mapped onto a noncritical one, ${ }^{8,9}$ with

$$
\begin{aligned}
& L_{r} \rightarrow \bar{L}_{r}(\lambda)=D_{L}\left(u_{r}\right) L_{r} e^{-\lambda / \nu}, \\
& n_{r} \rightarrow \bar{n}_{r}(\lambda)=n_{r} e^{d \lambda},
\end{aligned}
$$

where the dimensionless renormalized density correlation length ${ }^{44}$ is of order 1 , and with $u_{r} \rightarrow u_{r \text {.FP }}$ approaching the infrared-stable fixed point value $u_{r, \text { FP }}$. Here $D_{L}$ is a nonuniversal amplitude which depends on the strength $u_{r}$ of the excluded volume interaction. For the end-to-end distance $\mathcal{R}_{x}$ of a chain in dilute solution and for the osmotic pressure, the surface tension, and the coefficient of spontaneous curvature for arbitrary inter-chain overlap, the mapping yields ${ }^{8,9}$

$$
\begin{aligned}
& \mathcal{R}_{x}^{2}=\mu^{-2} \mathcal{X}\left(L_{r}, u_{r}\right)=\mu^{-2} e^{2 \lambda} \mathcal{X}\left(\bar{L}_{r}, u_{r, \mathrm{FP}}\right), \\
& \frac{\Pi}{n k_{B} T}=\mathcal{P}\left(n_{r}, L_{r}, u_{r}\right)=\mathcal{P}\left(\bar{n}_{r}, \bar{L}_{r}, u_{r, \mathrm{FP}}\right), \\
& \frac{\sigma}{n k_{B} T}=\mu^{-1} \mathcal{Q}\left(n_{r}, L_{r}, u_{r}\right)=\mu^{-1} e^{\lambda} \mathcal{Q}\left(\bar{n}_{r}, \bar{L}_{r}, u_{r, \mathrm{FP}}\right), \\
& \frac{\kappa}{n k_{B} T}=\mu^{-2} \mathcal{T}\left(n_{r}, L_{r}, u_{r}\right)=\mu^{-2} e^{2 \lambda} \mathcal{T}\left(\bar{n}_{r}, \bar{L}_{r}, u_{r, \mathrm{FP}}\right) .
\end{aligned}
$$

The noncritical manifold is chosen so that ${ }^{8,45}$

$$
\frac{\rho_{0}}{\bar{L}_{r}}+\zeta_{0} \bar{n}_{r} \bar{L}_{r}=1,
$$

where $\rho_{0}$ and $\zeta_{0}$ are constants ${ }^{46}$ of order 1 . The condition Eq. (3.10) interpolates ${ }^{44}$ between $\bar{L}_{r}=O(1)$ in the dilute limit and $\bar{L}_{r} /\left(\bar{n}_{r} \bar{L}_{r}^{2}\right) \propto \bar{L}_{r} / \overline{\mathcal{S}}=O(1)$ in the semidilute limit, i.e., the length scale is set by the end-to-end distance and the correlation or screening length, ${ }^{31}$ respectively. 
For the noncritical state the functions $\mathcal{X}, \mathcal{P}, \mathcal{Q}, \mathcal{T}$ can be approximated by their tree expressions

$$
\begin{aligned}
& \mathcal{X}\left(\bar{L}_{r}, u_{r, \mathrm{FP}}\right) \approx \mathcal{X}_{\text {tree }}\left(\bar{L}_{r}, u_{r, \mathrm{FP}}\right)=2 \bar{L}_{r}, \\
& \mathcal{P}\left(\bar{n}_{r}, \bar{L}_{r}, u_{r, \mathrm{FP}}\right) \approx \mathcal{P}_{\text {tree }}\left(\bar{n}_{r}, \bar{L}_{r}, u_{r, \mathrm{FP}}\right)=1+\frac{1}{2} a \bar{L}_{r}^{2} \bar{n}_{r} \\
& \mathcal{Q}\left(\bar{n}_{r}, \bar{L}_{r}, u_{r, \mathrm{FP}}\right) \approx \mathcal{Q}_{\text {tree }}\left(\bar{n}_{r}, \bar{L}_{r}, u_{r, \mathrm{FP}}\right) \\
& =\left(2 \bar{L}_{r}\right)^{1 / 2} g\left(a \bar{L}_{r}^{2} \bar{n}_{r}\right), \\
& \mathcal{T}\left(\bar{n}_{r}, \bar{L}_{r}, u_{r, \mathrm{FP}}\right) \approx \mathcal{T}_{\text {tree }}\left(\bar{n}_{r}, \bar{L}_{r}, u_{r, \mathrm{FP}}\right)=2 \bar{L}_{r} h\left(a \bar{L}_{r}^{2} \bar{n}_{r}\right),
\end{aligned}
$$

where

$$
a=\frac{1}{2}(4 \pi)^{3 / 2} u^{*}=8.11
$$

in $d=3$. Here $a L_{r}^{2} n_{r}$ follows from $\mathcal{S}=b N^{2} n$ in Eq. (2.3) on using the reparameterization Eqs. (3.1)-(3.3), dropping the ensuing $Z$-factors $Z_{u} / Z_{t}^{2}$ which is consistent within the tree approximation, and using the fixed-point value $u_{r, \mathrm{FP}}=\frac{3}{2} u^{*}$ with $u^{*}=0.364$ in $d=3$ dimensions, given in Eq. (13.4) of Ref. 8. The functions $g$ and $h$ are our mean-field scaling functions from Eqs. (2.31), (2.32), and Fig. 5 and from Eqs. (2.34), (2.35), and Fig. 6.

For the end-to-end distance $\mathcal{R}_{x}$ of a chain in a dilute solution, $n_{r}$ vanishes, and the flow condition Eq. (3.10) becomes $\bar{L}_{r}=\rho_{0}$. Thus Eq. (3.4) leads to $e^{2 \lambda}=\left(D_{L} L_{r} / \rho_{0}\right)^{2 \nu}$, and Eqs. (3.6) and (3.11) to the relation

$$
\frac{1}{2}\left(\mu \mathcal{R}_{x}\right)^{2}=\rho_{0}^{1-2 \nu}\left(D_{L} L_{r}\right)^{2 \nu} .
$$

We now evaluate the scaling functions for the osmotic pressure, the surface tension, and the coefficient of spontaneous curvature. Here it is advantageous to use the first term in Eq. (3.10),

$$
\frac{\rho_{0}}{\bar{L}_{r}}=v
$$

as an intermediate variable ${ }^{8} v$. Note that $\lambda$ drops out of the combination,

$$
\begin{aligned}
(1-v) / v^{d \nu-1} & =\zeta_{0} \rho_{0}^{-d \nu+1} n_{r}\left(D_{L} L_{r}\right)^{d \nu} \\
& =\zeta_{0} \rho_{0}^{-(d / 2)+1} n\left(\mathcal{R}_{x} / \sqrt{2}\right)^{d} .
\end{aligned}
$$

In the last step Eqs. (3.16) and (3.3) have been used. In three dimensions the mean square radius of gyration $\mathcal{R}_{g}^{2} \equiv 3 \mathcal{R}_{g, x}^{2}$ is equal to $\mathcal{R}_{x}^{2} / 2$, to a very good approximation, ${ }^{7}$ and the usual geometrical overlap $s_{3} \equiv s$ equals

$$
s \equiv n \mathcal{R}_{g}^{3} \approx n\left(\mathcal{R}_{x} / \sqrt{2}\right)^{3} \equiv s_{3}^{(x)} /(2 \sqrt{2}) .
$$

Thus, in three dimensions the intermediate variable Eq. (3.17) is related to the geometrical overlap via

$$
(1-v) / v^{3 \nu-1}=\zeta_{0} \rho_{0}^{-1 / 2} s .
$$

Since

$$
a \bar{L}_{r}^{2} \bar{n}_{r}=\frac{a \rho_{0}}{\zeta_{0}}\left(\frac{1}{v}-1\right),
$$

one finds from Eqs. (3.7), (3.12) the osmotic pressure,

$$
\frac{\Pi}{n k_{B} T}=1+\frac{1}{2} \frac{a \rho_{0}}{\zeta_{0}}\left(\frac{1}{v}-1\right) .
$$

For the surface tension in Eqs. (3.8), (3.13) and the coefficient of spontaneous curvature in Eqs. (3.9), (3.14), we also need

$$
\left(2 \bar{L}_{r}\right)^{1 / 2}=\left(2 \rho_{0}\right)^{1 / 2} v^{-1 / 2},
$$

which follows from Eq. (3.17), and

$$
\mu^{-1} e^{\lambda}=\mu^{-1}\left(v \frac{D_{L} L_{r}}{\rho_{0}}\right)^{\nu}=v^{\nu}\left(2 \rho_{0}\right)^{-1 / 2} \mathcal{R}_{x},
$$

where Eqs. (3.17), (3.4) and Eq. (3.16) have been used in the last two steps. This leads to

$$
\frac{\sigma}{n k_{B} T}=\mathcal{R}_{x} v^{\nu-1 / 2} g\left(\frac{a \rho_{0}}{\zeta_{0}}\left(\frac{1}{v}-1\right)\right)
$$

and

$$
\frac{\kappa}{n k_{B} T}=\mathcal{R}_{x}^{2} v^{2 \nu-1} h\left(\frac{a \rho_{0}}{\zeta_{0}}\left(\frac{1}{v}-1\right)\right) .
$$

In the special cases of small and large overlap Eq. (3.20) implies

$$
\begin{aligned}
& \frac{\Pi}{n k_{B} T} \rightarrow\left(1+\frac{1}{2} a \rho_{0}^{1 / 2} s, \quad \frac{1}{2} \frac{a \rho_{0}}{\zeta_{0}}\left(\frac{\zeta_{0} s}{\rho_{0}^{1 / 2}}\right)^{1 /(3 \nu-1)}\right), \\
& s \rightarrow(0, \infty)
\end{aligned}
$$

for the osmotic pressure in Eq. (3.22),

$$
\begin{aligned}
\frac{\sigma}{n k_{B} T \mathcal{R}_{x}} \rightarrow & \left(\sqrt{\frac{2}{\pi}}\left(1+\left[a \rho_{0}^{1 / 2} \lambda_{1}-\zeta_{0} \rho_{0}^{-1 / 2}\left(\nu-\frac{1}{2}\right)\right] s\right),\right. \\
& \left.\frac{2}{3}\left(\frac{a \rho_{0}}{\zeta_{0}}\right)^{1 / 2}\left(\frac{\zeta_{0} s}{\rho_{0}^{1 / 2}}\right)^{(1-\nu) /(3 \nu-1)}\right), \quad s \rightarrow(0, \infty)
\end{aligned}
$$

for the surface tension in Eq. (3.25), and

$$
\begin{aligned}
\frac{\kappa}{n k_{B} T \mathcal{R}_{x}^{2}} \rightarrow & \left(\frac{1}{2}\left(1+\left[2 a \rho_{0}^{1 / 2} \lambda_{2}-\zeta_{0} \rho_{0}^{-1 / 2}(2 \nu-1)\right] s\right),\right. \\
& \left.\frac{1}{3}(4 \ln 2-1)\left(\frac{\zeta_{0} s}{\rho_{0}^{1 / 2}}\right)^{-(2 \nu-1) /(3 \nu-1)}\right), \quad s \rightarrow(0, \infty)
\end{aligned}
$$

for the coefficient of spontaneous curvature. The exponents $1 /(3 \nu-1),(1-\nu) /(3 \nu-1)$, and $-(2 \nu-1) /(3 \nu-1)$ indicate that $\Pi / k_{B} T, \sigma / k_{B} T$, and $\kappa / k_{B} T$ in the limit of strong overlap only depend on the monomer density, i.e., only on the combination $n \mathcal{R}_{x}^{1 / \nu}$, and are proportional to $\xi^{-3}, \xi^{-2}$, and $\xi^{-1}$, respectively. The values of the two constants,

$$
\rho_{0}=1.81, \quad \zeta_{0}=10.9
$$

follow from comparing Eq. (3.27) with known amplitude ratios of the osmotic pressure. , $^{8,7}$

Figure 17 shows the surface tension which follows from Eqs. (3.25) and (3.20). The limiting behavior for small and large overlap is given by 


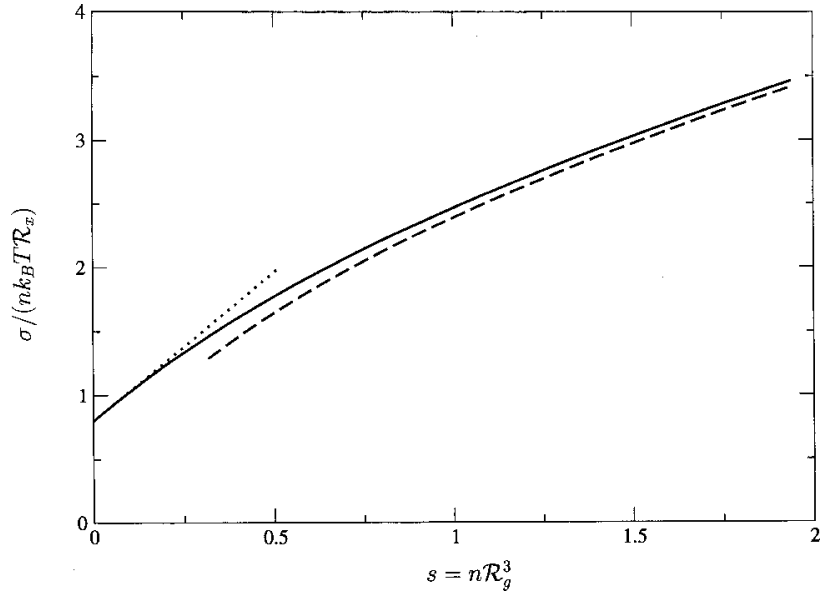

FIG. 17. Scaling function of the surface tension in the renormalized tree approximation [Eqs. (3.25), (3.20)]. The quantity $s$ is the geometrical overlap in Eq. (3.19). The dotted and dashed lines show the asymptotic behavior for small and large $s$, respectively [compare Eq. (3.31)].

$$
\begin{aligned}
\frac{\sigma}{n k_{B} T \mathcal{R}_{x}} \rightarrow & \left(0.798\left[1+1.032 n \mathcal{R}_{x}^{3}\right],\right. \\
& \left.\left(1.781 n \mathcal{R}_{x}^{3}\right)^{0.539}\right), \quad s \rightarrow(0, \infty) .
\end{aligned}
$$

In Eq. (3.31) the leading contribution in the dilute limit equals the result for ideal chains. Actually in an $\epsilon$-expansion one finds a small correction $0.798 \rightarrow 0.798(1-0.051 \epsilon)$ due to monomer-monomer repulsion. ${ }^{16}$ The value 1.781 of the universal amplitude in the semidilute limit is not too far from the extrapolation 2.19 to three dimensions of the leading contribution $2 \pi^{2} \epsilon / 9$ in the $\epsilon$-expansion. The leading contribution near four dimensions follows from inserting the fixed point value Eqs. (2.4), (2.3) into the mean-field expression Eqs. (2.32) and (2.31).

Figure 18 shows the coefficient of spontaneous curvature which follows from Eqs. (3.26) and (3.20). In the case of the coefficient of spontaneous curvature the mean-field result (Fig. 6) and the result from the renormalized tree approximation (Fig. 18) are qualitatively different. Note that the

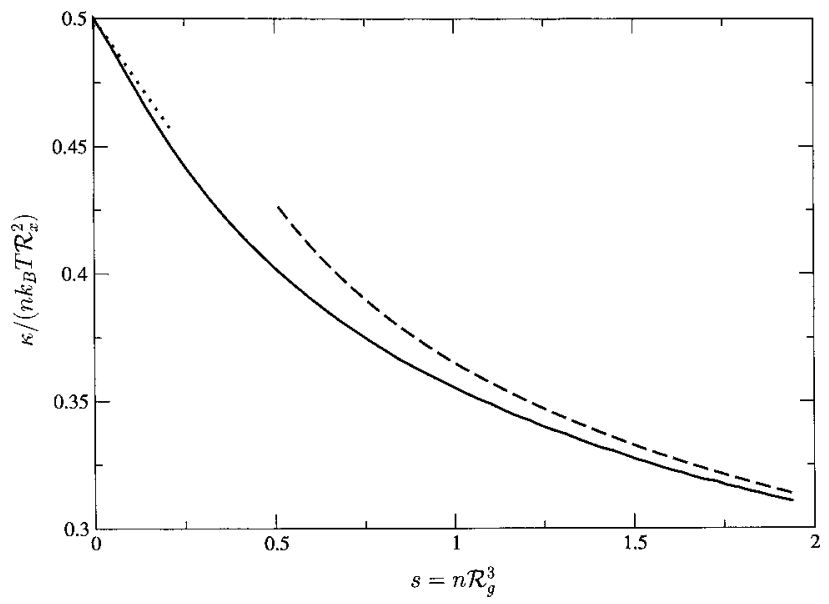

FIG. 18. Scaling function of the coefficient of spontaneous curvature in the renormalized tree approximation [Eqs. (3.26), (3.20)]. The dotted and dashed lines show the asymptotic behavior for small and large geometrical overlap $s$, respectively [compare Eq. (3.29)]. overlap-dependence $s^{-(2 \nu-1) /(d \nu-1)}$ for $s \rightarrow \infty$ leads to a constant in the mean-field approximation (with $\nu=1 / 2$ ), while it leads to a power-law decay in the renormalized tree approximation (with $\nu=0.588$ ).

\section{SUMMARY AND CONCLUDING REMARKS}

We have studied the solvation free energy and the polymer density depletion profile of a single mesoscopic colloidal particle immersed in a solution of free nonadsorbing polymer chains. Our main goal was to give a global description valid for arbitrary values of the particle to polymer size ratio $\rho=R / \mathcal{R}_{x}$ and of the inter-chain overlap $\mathcal{S}=n / n^{*}$. It is interesting to see how the qualitatively different behavior evolves in the limits of small and large size ratio and of a dilute and a semidilute polymer solution.

Most of our results have been obtained within a meanfield description of the polymer solution; see Eqs. (2.8)(2.13). While this is quantitatively correct only near four dimensions, ${ }^{22}$ most of the qualitative features persist down to three dimensions. The mean-field approximation is a consistent theory, which obeys exact relationships such as the density-pressure relation [see Eqs. (1.2), (1.3) and (2.22), (2.23)] and identities which follow from the small radius expansion (see Secs. IIF and II G). Our mean-field results obtained for a cylindrical particle in four dimensions can be used to describe the qualitative features of a spherical particle in three dimensions (see Sec. II B). More quantitative results in three dimensions were obtained for the surface tension of a planar boundary and for the coefficient of spontaneous curvature in a Helfrich expansion by means of a "renormalized tree approximation" (see Sec. III).

Here is a summary of our main results.

(1) Scaling functions for the free energy of immersion of a particle and for the pressure which the polymers exert on the particle. Our numerical mean-field results in Figs. 2 and 3 interpolate smoothly between the analytical results for small [Eqs. (1.5) and (1.2)] and large [Eqs. (2.28) and (2.29)] size ratio $\rho$. Note that the results for small $\rho$ are independent of the inter-chain overlap $\mathcal{S}$. For increasing $\rho$ the pressure decreases, due to an entropically driven decrease in the polymer density near a particle of increasing size.

(2) Scaling function for the decrease of the number of chains $\langle-\delta \mathcal{N}\rangle$ on immersing a particle: In the limits of large and small size ratios $\rho$ we obtained the analytic expressions in Eqs. (2.38) and (2.44), respectively. Our numerical results in Fig. 4 interpolate smoothly between these limits.

(3) The density-pressure identity in Eqs. (2.22), (2.23) within the mean-field approximation has been derived in Appendix A. In order to check the accuracy of our numerical procedure, we compare, in Figs. 7 and 8, the amplitude of the polymer density profile near the surface with the pressure for the case of a planar wall and of a weakly curved particle surface, respectively. The overlap-dependence of both the bulk osmotic pressure and the surface tension can be identified with high accuracy in the density amplitude. An analytic check for the semidilute limit is presented in Eq. (E12) of Appendix E. The case of a small particle radius is discussed in the paragraph preceding Eq. (2.44). 
(4) Shapes of the density profiles $\mathcal{M}$ : Here we find that one has to distinguish between gross and fine-structure effects. The gross form of $\mathcal{M}\left(r_{\perp}\right)$ is a function which increases monotonically with increasing $r_{\perp}$ and has a point of inflection at $r_{\perp}-R=\xi_{I}$ (see Figs. 10, 11, and 14). While for $R \gg \mathcal{R}_{x}$ the scaled distance of the point of inflection $\xi_{I} / \mathcal{R}_{x}$ from the particle surface decreases with increasing overlap (roughly in the same way as the density correlation length $\left.\xi_{D}\right)$, for $R \ll \mathcal{R}_{x}, \xi_{D}$ it is of the order of $R / \mathcal{R}_{x}$, i.e., independent of the overlap. On a finer scale we find a maximum of $\mathcal{M}$ (see Fig. 12). In the case of a planar wall similar maxima have been reported in Refs. 40, 41. We find that the maximum persists down to small size ratio $R \ll \mathcal{R}_{x}$ and is always largest for $\mathcal{S} \approx 5$. Finally we have established that for the case $R \ll \mathcal{R}_{x}, \xi_{D}$ in which the cylindrical (spherical) particle acts as a line (point) perturbation on the polymer solution, the maximum can be understood in terms of a minimum ${ }^{42}$ of the density-density correlation function of the pure polymer solution; see Eq. (2.41) and Fig. 16. These single-particle results imply conclusions for the interaction between two particles: The r-dependence of the free energy cost of immersing a small spherical particle at point $\mathbf{r}$ in the neighborhood of our particle or wall is proportional to the r-dependence of the single-particle density profile $\mathcal{M}$.

(5) Density profile for small particle radius in the semidilute limit: The full curve in Fig. 9 shows the density profile for the length ratios $R: \xi: \mathcal{R}_{x}=1: 10: 100$ which corresponds to a large overlap of $\mathcal{S}=25$. Here the density crosses over from the $\xi$-independent power law behavior for $R, r_{\perp} \ll \xi$ given in Eq. (1.4) to an exponential decay toward the bulk value for $R \ll r_{\perp}, \xi$ which is ruled by the screening length $\xi$; see Eqs. (E16) and (E17).

(6) Surface tension and coefficient of spontaneous curvature: In the Helfrich expansion Eq. (2.28) for a weakly curved particle surface we have evaluated the scaling functions for the overlap dependence of the surface tension $\sigma$ and of the coefficient $\kappa$ of spontaneous curvature both in the mean field and renormalized tree approaches. In the case of $\sigma$ the mean-field result in Eqs. (2.30), (2.31), (2.32), and Fig. 5 agrees qualitatively with the renormalized tree result in Eqs. (3.25), (3.20), (3.28), (3.31), and Fig. 17. However, there are qualitative differences in the case of $\kappa$, as can be seen by comparing the mean-field result in Fig. 6 with the renormalized tree prediction in Fig. 18. In particular the scaling law $\kappa /\left(n \mathcal{R}_{x}^{2}\right) \propto s^{-(2 \nu-1) /(d \nu-1)}$ in the semidilute limit [which guarantees that $\kappa$ only depends on the combination $n \mathcal{R}_{x}^{1 / \nu}$ of the segment density and is proportional to $\xi^{-d+2}$ ] leads to an $s$-independent behavior in the mean-field approximation (with $\nu=1 / 2$ ) and to a power law decay in the overlap $s$ in the renormalized tree approximation (with $\nu$ $=0.588$ ).

\section{ACKNOWLEDGMENT}

It is a pleasure to thank T. W. Burkhardt for useful discussions.

\section{APPENDIX A: DENSITY-PRESSURE IDENTITY}

\section{Pressure on a sphere from a single ideal chain}

The partition function $Z^{[0]}\left(L ; \mathbf{r}_{A}, \mathbf{r}_{B} ; R\right)$ of an ideal chain with ends fixed at $\mathbf{r}_{A}$ and $\mathbf{r}_{B}$ outside a spherical particle with radius $R$ satisfies the diffusionlike equation (2.8) with $L^{\prime}, \mathbf{r}, \mathcal{V}$ replaced by $L, \mathbf{r}_{A}, 0$ and the "initial condition" Eq. (2.10) replaced by $Z^{[0]}\left(L=0 ; \mathbf{r}_{A}, \mathbf{r}_{B} ; R\right)=\delta\left(\mathbf{r}_{A}-\mathbf{r}_{B}\right)$. It is useful to introduce the Laplace transform

$$
\begin{aligned}
G_{S}\left(t ; \mathbf{r}_{A}, \mathbf{r}_{B}\right) & =\int_{0}^{\infty} d L e^{-L t} Z^{[0]}\left(L ; \mathbf{r}_{A}, \mathbf{r}_{B} ; R\right) \\
& \equiv \mathcal{L} Z^{[0]}\left(L ; \mathbf{r}_{A}, \mathbf{r}_{B} ; R\right),
\end{aligned}
$$

which is a correlation function ${ }^{48}\left\{\phi\left(\mathbf{r}_{A}\right) \phi\left(\mathbf{r}_{B}\right)\right\}_{S}$ of a Gaussian Ginzburg-Landau model ${ }^{6-9}$ and satisfies an OrnsteinZernike type equation

$$
\left(-\Delta_{r_{A}}+t\right) G_{S}\left(t ; \mathbf{r}_{A}, \mathbf{r}_{B}\right)=\delta\left(\mathbf{r}_{A}-\mathbf{r}_{B}\right) .
$$

Both $Z^{[0]}$ and $G_{S}$ vanish on the surface $S$ of the spherical particle, which is centered at the origin.

Now consider a particle with a surface $S^{\prime}$ which deviates slightly from the spherical surface $S . S^{\prime}$ is obtained by shifting each surface point $\mathbf{r}_{S}$ of $S$ by a small amount $\eta\left(\Omega_{S}\right)$ toward the center of $S$. Here $\Omega_{S}$ is the solid angle of the surface point $\mathbf{r}_{S}$. For the particular cases $\eta\left(\Omega_{S}\right)=$ const or $\eta\left(\Omega_{S}\right) \propto \cos \vartheta_{S}$, the surface $S^{\prime}$ is also spherical, but, compared to $S$, its radius is decreased $\left(R^{\prime}=R-\eta\right)$ or its center is shifted along the polar axis. To first order in the small deviation $\eta$, the correlation function $G_{S^{\prime}}$ for the deformed surface $S^{\prime}$ is related to correlation functions for the nondeformed spherical surface $S$ via

$$
\begin{aligned}
G_{S^{\prime}}\left(t ; \mathbf{r}_{A}, \mathbf{r}_{B}\right)= & G_{S}\left(t ; \mathbf{r}_{A}, \mathbf{r}_{B}\right)+\int d S \eta\left(\Omega_{S}\right) \\
& \times\left\{\frac{1}{2}\left(\partial_{n} \phi\left(\mathbf{r}_{S}\right)\right)^{2} \cdot \phi\left(\mathbf{r}_{A}\right) \phi\left(\mathbf{r}_{B}\right)\right\}_{S} .
\end{aligned}
$$

Here $\partial_{n}$ is a derivative perpendicular to $S$, and

$$
\begin{aligned}
& \left\{\frac{1}{2}\left(\partial_{n} \phi\left(\mathbf{r}_{S}\right)\right)^{2} \cdot \phi\left(\mathbf{r}_{A}\right) \phi\left(\mathbf{r}_{B}\right)\right\}_{S} \\
& \quad=\left\{\partial_{n} \phi\left(\mathbf{r}_{S}\right) \phi\left(\mathbf{r}_{A}\right)\right\}_{S}\left\{\partial_{n} \phi\left(\mathbf{r}_{S}\right) \phi\left(\mathbf{r}_{B}\right)\right\}_{S},
\end{aligned}
$$

due to Wick's theorem. Obviously $G_{S^{\prime}}$ in Eq. (A3) satisfies Eq. (A2) for arbitrary points $\mathbf{r}_{A}, \mathbf{r}_{B}$ off the surface. As we show below,

$$
G_{S^{\prime}}\left(t ; \mathbf{r}_{A}, \mathbf{r}_{B}\right) \rightarrow\left[r_{A}-\left(R-\eta\left(\Omega_{A}\right)\right)\right]\left\{\partial_{n} \phi\left(\mathbf{r}_{A}\right) \phi\left(\mathbf{r}_{B}\right)\right\}_{S}
$$

as $\mathbf{r}_{A}$ approaches the surface and $\mathbf{r}_{B}$ is off the surface. Thus $G_{S^{\prime}}$ vanishes at the deformed surface $S^{\prime}$.

To derive Eq. (A5) we use the explicit form of $G_{S}$, which for a sphere in $d$ dimensions is given by ${ }^{13,16}$

$$
\begin{aligned}
G_{S}\left(t ; \mathbf{r}_{A}, \mathbf{r}_{B}\right) & \equiv\left\{\phi\left(\mathbf{r}_{A}\right) \phi\left(\mathbf{r}_{B}\right)\right\}_{S} \\
& =\sum_{l=0}^{\infty} W_{l}^{(\alpha)}(\vartheta) \hat{G}_{l}\left(t ; \mathbf{r}_{A}, \mathbf{r}_{B} ; R\right) .
\end{aligned}
$$

Here $\alpha=(d-2) / 2, \vartheta$ is the angle between $\mathbf{r}_{A}$ and $\mathbf{r}_{B}$, and

$$
W_{l}^{(\alpha)}=\left(2 \pi^{d / 2}\right)^{-1} \Gamma(\alpha)(l+\alpha) C_{l}^{(\alpha)}(\cos \vartheta),
$$


where $C_{l}^{(\alpha)}$ are Gegenbauer polynomials, and

$$
\begin{aligned}
\hat{G}_{l}= & \left(r_{<} r_{>}\right)^{-\alpha} K_{\alpha+l}\left(\sqrt{t} r_{>}\right)\left(I_{\alpha+l}\left(\sqrt{t} r_{<}\right)\right. \\
& \left.-\frac{I_{\alpha+l}(\sqrt{t} R)}{K_{\alpha+l}(\sqrt{t} R)} K_{\alpha+l}\left(\sqrt{t} r_{<}\right)\right)
\end{aligned}
$$

where $r_{<}=\min \left(r_{A}, r_{B}\right), r_{>}=\max \left(r_{A}, r_{B}\right)$ and $I, K$ are modified Bessel functions. This implies

$$
\begin{aligned}
& \left\{\left(\partial_{n} \phi\left(\mathbf{r}_{S}\right)\right) \phi\left(\mathbf{r}_{A}\right)\right\}_{S} \\
& \quad=\sum_{l=0}^{\infty} W_{l}^{(\alpha)}\left(\vartheta_{\mathbf{r}_{S}, \mathbf{r}_{A}}\right) R^{-1-\alpha} r_{A}^{-\alpha} K_{\alpha+l}\left(\sqrt{t} r_{A}\right) / K_{\alpha+l}(\sqrt{t} R) .
\end{aligned}
$$

Equation (A5) now follows since Eq. (A9) for $r_{A} \rightarrow R$ becomes a $\delta$-function in the solid angle, i.e., ${ }^{49}$

$$
\lim _{r_{A} \rightarrow R} R^{d-1} \int d \Omega_{S} f\left(\Omega_{S}\right)\left\{\left(\partial_{n} \phi\left(\mathbf{r}_{S}\right)\right) \phi\left(\mathbf{r}_{A}\right)\right\}_{S}=f\left(\Omega_{A}\right)
$$

for arbitrary smooth test functions $f$. While $\left\{\left(\partial_{n} \phi\left(\mathbf{r}_{S}\right)\right) \phi\left(\mathbf{r}_{A}\right)\right\}_{S}$ vanishes because of the Dirichlet condition if $\mathbf{r}_{A}$ approaches a point on the surface $S$ which is different from $\mathbf{r}_{S}$, the Dirichlet condition is broken for $\mathbf{r}_{A} \rightarrow \mathbf{r}_{S}$ by the operator $\partial_{n} \phi\left(\mathbf{r}_{S}\right)$.

With the help of Eq. (A3) one can express the change in free energy of a polymer with two fixed ends on deforming the particle surface

$$
\frac{F_{S^{\prime}}-F_{S}}{k_{B} T}=-\ln \frac{\mathcal{L}^{-1} G_{S^{\prime}}}{\mathcal{L}^{-1} G_{S}}=-\int d S \eta\left(\Omega_{S}\right) \frac{p(S)}{k_{B} T}
$$

in terms of the local polymer pressure $p(S)$ which acts on a surface element $d S$ of the nondeformed spherical surface $S$. Here $\mathcal{L}^{-1}$ is the inverse of the Laplace transform in Eq. (A1). Since the two ends are fixed at $\mathbf{r}_{A}, \mathbf{r}_{B}$,

$$
\begin{aligned}
\frac{p(S)}{k_{B} T} & =\frac{p\left(\mathbf{r}_{S} ; \mathbf{r}_{A}, \mathbf{r}_{B}\right)}{k_{B} T} \\
& =\frac{\mathcal{L}^{-1}\left\{\frac{1}{2}\left(\partial_{n} \phi\left(\mathbf{r}_{S}\right)\right)^{2} \cdot \phi\left(\mathbf{r}_{A}\right) \phi\left(\mathbf{r}_{B}\right)\right\}_{S}}{\mathcal{L}^{-1}\left\{\phi\left(\mathbf{r}_{A}\right) \phi\left(\mathbf{r}_{B}\right)\right\}_{S}} .
\end{aligned}
$$

If only one end is fixed at $\mathbf{r}_{A}$ and the other end is free,

$$
\begin{aligned}
\frac{p(S)}{k_{B} T} & =\frac{p\left(\mathbf{r}_{S} ; \mathbf{r}_{A}\right)}{k_{B} T} \\
& =\frac{\mathcal{L}^{-1}\left\{\frac{1}{2}\left(\partial_{n} \phi\left(\mathbf{r}_{S}\right)\right)^{2} \cdot \phi\left(\mathbf{r}_{A}\right) \int d \mathbf{r}_{B} \phi\left(\mathbf{r}_{B}\right)\right\}_{S}}{\mathcal{L}^{-1}\left\{\phi\left(\mathbf{r}_{A}\right) \int d \mathbf{r}_{B} \phi\left(\mathbf{r}_{B}\right)\right\}_{S}} .
\end{aligned}
$$

A simple explicit result follows for a long chain with one end fixed at $\mathbf{r}_{A}$ outside the sphere. In this case $R, r_{A} \ll \mathcal{R}_{x}$, the Bessel functions in Eq. (A9) can be expanded for small argument, and one finds

$$
\frac{p\left(\mathbf{r}_{S} ; \mathbf{r}_{A}\right)}{k_{B} T}=\frac{(d-2) \Gamma(d / 2)}{2 \pi^{d / 2}} \frac{\left(r_{A} / R\right)^{2}-1}{1-\left(R / r_{A}\right)^{d-2}} \frac{1}{\left|\mathbf{r}_{A}-\mathbf{r}_{S}\right|^{d}} .
$$

In $d=3$ the derivative of the polymer free energy with respect to the radius of the sphere is given by

$$
\int d S \frac{p\left(\mathbf{r}_{S} ; \mathbf{r}_{A}\right)}{k_{B} T}=\frac{1}{r_{A}-R}
$$

and the repulsive force between the fixed point and the sphere by

$$
\int d S \cos \vartheta_{\mathbf{r}_{S}, \mathbf{r}_{A}} \frac{p\left(\mathbf{r}_{S} ; \mathbf{r}_{A}\right)}{k_{B} T}=\frac{R / r_{A}}{r_{A}-R} .
$$

Both results are consistent with the free energy cost,

$$
\frac{F_{S}}{k_{B} T}=-\ln \left(1-\frac{R}{r_{A}}\right)
$$

of introducing the spherical obstacle $S$.

The pressure in Eqs. (A12) and (A13) is related to the density of polymer material near the surface point $\mathbf{r}_{S}$. For example for an ideal chain with two ends fixed at $\mathbf{r}_{A}$ and $\mathbf{r}_{B}$, the fraction of monomers,

$$
\vartheta(\mathbf{r}) d \mathbf{r} \equiv \frac{1}{N} \sum_{j=1}^{N} \delta\left(\mathbf{r}-\mathbf{r}_{j}\right) d \mathbf{r},
$$

in a volume element $d \mathbf{r}$ is related to the partition function $Z^{[\mathcal{W}]}$ of a chain subject to an external potential $\mathcal{W}$ by

$$
\frac{\mathcal{R}_{x}^{2}}{2}\langle\vartheta(\mathbf{r})\rangle_{A, B}=-\left(\frac{\delta}{\delta \mathcal{W}(\mathbf{r})} \ln Z^{[\mathcal{W}]}\left(L ; \mathbf{r}_{A}, \mathbf{r}_{B} ; R\right)\right)_{\mathcal{W}=0} .
$$

Here $Z^{[\mathcal{W}]}$ satisfies the diffusionlike equation (2.8) with $L^{\prime}, \mathbf{r}, \mathcal{V}$ replaced by $L, \mathbf{r}_{A}, \mathcal{W}$. The derivative of $Z^{[\mathcal{W}]}$ is related by

$$
\begin{array}{r}
-\left(\frac{\delta}{\delta \mathcal{W}(\mathbf{r})} Z^{[\mathcal{W}]}\left(L ; \mathbf{r}_{A}, \mathbf{r}_{B} ; R\right)\right)_{\mathcal{W}=0} \\
=\mathcal{L}^{-1}\left\{\frac{1}{2} \phi^{2}(\mathbf{r}) \cdot \phi\left(\mathbf{r}_{A}\right) \phi\left(\mathbf{r}_{B}\right)\right\}_{S}
\end{array}
$$

to the Ginzburg-Landau correlation function with $\phi^{2}$ inserted. When $\mathbf{r}$ approaches the surface $\mathbf{r}_{S}$ of the spherical particle,

$$
\frac{1}{2} \phi^{2}(\mathbf{r}) \rightarrow(r-R)^{2} \frac{1}{2}\left(\partial_{n} \phi\left(\mathbf{r}_{S}\right)^{2},\right.
$$

and Eq. (A12) leads to the density-pressure relation,

$$
\left\langle\vartheta\left(\mathbf{r} \rightarrow \mathbf{r}_{S}\right)\right\rangle_{A, B} \rightarrow 2 \frac{(r-R)^{2}}{\mathcal{R}_{x}^{2}} \frac{p\left(\mathbf{r}_{S} ; \mathbf{r}_{A}, \mathbf{r}_{B}\right)}{k_{B} T} .
$$

For later use we record the relation

$$
\begin{aligned}
-\frac{d}{d R} Z^{[0]}\left(L ; \mathbf{r}_{A}, \mathbf{r}_{B} ; R\right) & \\
= & \int d S\left(-\frac{\delta}{\delta \mathcal{W}(\mathbf{r})} Z^{[\mathcal{W}]}\left(L ; \mathbf{r}_{A}, \mathbf{r}_{B} ; R\right) /\right. \\
& \left.(r-R)^{2}\right)_{\mathbf{r} \rightarrow \mathbf{r}_{S}, \mathcal{W}=0},
\end{aligned}
$$




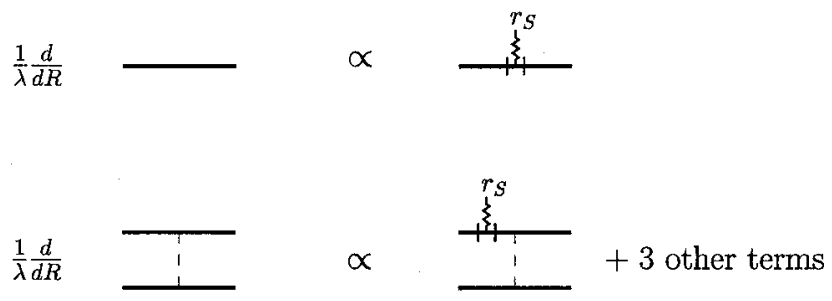

FIG. 19. Diagrammatic representation of the derivative of the free energy cost with respect to the radius of the cylindrical particle [Eqs. (A23), (A24)]. Each diagram has its counterpart in the fugacity expansion Eq. (A25) of the polymer density near the surface.

which follows from Eq. (A3) with an angular-independent $\eta=-d R$ and from Eqs. (A20) and (A21).

The relations Eqs. (A3), (A5), (A10)-(A13) and (A23) can be generalized in an obvious way to other surfaces $S$ such as cylinders or ellipsoids. In particular, Eq. (A23) applies to a cylinder of radius $R$ and infinite length if $r$ is replaced by the distance $r_{\perp}$ of point $\mathbf{r}$ from the axis of the cylinder.

\section{Density-pressure identity for mutually repelling chains in the mean-field approximation}

To derive Eq. (1.3) it is convenient to use the grand canonical ensemble. The derivative of the free energy for a cylinder in Eq. (1.2) is given by

$$
\frac{d}{d R} \frac{F / \lambda}{k_{B} T}=\sum_{\mathcal{N}=1}^{\infty} \frac{\zeta^{\mathcal{N}}}{\mathcal{N} !}\left[-\frac{d}{d R} \mathcal{Z}_{c}^{(\mathcal{N})} / \lambda\right]
$$

and the density profile of free polymers by

$$
\begin{aligned}
n \mathcal{M}\left(r_{\perp}\right)=\langle\Theta(\mathbf{r})\rangle & \equiv\left\langle\sum_{P=1}^{\mathcal{N}} \vartheta_{P}(\mathbf{r})\right\rangle \\
& =\frac{1}{L} \sum_{\mathcal{N}=1}^{\infty} \frac{\zeta^{\mathcal{N}}}{\mathcal{N} !}\left(-\frac{\delta}{\delta \mathcal{W}(\mathbf{r})} \mathcal{Z}_{c}^{(\mathcal{N})[\mathcal{W}]}\right)_{\mathcal{W}=0} .
\end{aligned}
$$

Here $\vartheta_{P}=(1 / N) \sum_{j=1}^{N} \delta\left(\mathbf{r}-\mathbf{r}_{P, j}\right)$, and $\mathcal{Z}_{c}^{(\mathcal{N})}$ is the connected part of the partition function of $\mathcal{N}$ chains with monomermonomer interaction in the tree approximation. There is an obvious correspondence between tree diagrams of Eq. (A24) shown in Fig. 19 and tree diagrams (with r-insertions) of Eq. (A25). Using Eq. (A23) for each ideal-polymer line in Eq. (A24) generates all the corresponding diagrams of Eq. (A25), with prefactors such that Eq. (1.3) holds in the form of Eq. (2.22). ${ }^{50}$

\section{APPENDIX B: AUXILIARY FIELD AND MEAN-FIELD APPROXIMATION}

The excluded volume interaction between monomers is the volume integral of the square of the monomer density operator. The square may be linearized by introducing a fluctuating potential field $\mathcal{W}$ with a Gaussian weight, and the mean-field approximation is obtained on replacing functional integration over $\mathcal{W}$ by taking the extremum of the new "ac- tion" $\mathcal{A}$ with respect to $\mathcal{W}$. If the extremum occurs at $\mathcal{W}$ $=\mathcal{V}$, the grand-canonical polymer free energy $\mathcal{F}_{G}$ per $k_{B} T$ in mean-field approximation is given by

$$
\mathcal{F}_{G}=\mathcal{A}[\mathcal{V}],
$$

where

$$
\mathcal{A}[\mathcal{W}]=\int d \mathbf{r}^{\prime}\left(-\zeta Z^{[\mathcal{W}]}\left(L, \mathbf{r}^{\prime}\right)-\frac{l^{4}}{2 b} \mathcal{W}^{2}\left(\mathbf{r}^{\prime}\right)\right),
$$

and

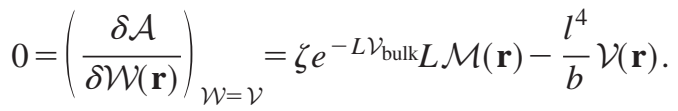

Here we consider a polymer solution with an embedded particle inside a large but finite volume $\mathcal{U}$. The spatial integration in Eq. (B2) is over that part of $\mathcal{U}$ which is outside the volume $V$ occupied by the particle. Apart from layers with a width of the order of the correlation length around the particle and the boundaries of $\mathcal{U}$, the solution shows bulk behav-

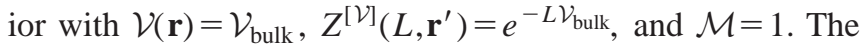
chain fugacity $\zeta$ may be eliminated in favor of the chain density, yielding

$$
\begin{aligned}
n & =-\zeta \frac{d}{d \zeta} \frac{\mathcal{F}_{G}}{\mathcal{U}}=\zeta \frac{d}{d \zeta}\left(\zeta e^{-L \mathcal{V}_{\text {bulk }}+\frac{l^{4}}{2 b}} \mathcal{V}_{\text {bulk }}^{2}\right) \\
& =\zeta e^{-L \mathcal{V}_{\text {bulk }}}
\end{aligned}
$$

Note that the $\zeta$-dependence of $\mathcal{V}_{\text {bulk }}$ does not contribute to the derivative, due to the extremum condition Eq. (B3). Substituting Eq. (B4) into Eq. (B3) shows that

$$
\mathcal{V}(\mathbf{r})=\frac{n L b}{l^{4}} \mathcal{M}(\mathbf{r})=\frac{\mathcal{S}}{L} \mathcal{M}(\mathbf{r}),
$$

i.e., the potential field at the extremum is identical with the mean-field Eq. (2.9), and

$$
L \mathcal{V}_{\text {bulk }}=\mathcal{S} \text {. }
$$

The first term on the right hand side of Eq. (B2) can also be expressed in terms of the density profile $\mathcal{M}$, since due to the chain structure

$$
\int d \mathbf{r}^{\prime} Z^{[\mathcal{V}]}\left(L, \mathbf{r}^{\prime}\right)=\int d \mathbf{r} Z^{[\mathcal{V}]}\left(L^{\prime \prime}, \mathbf{r}\right) Z^{[\mathcal{V}]}\left(L-L^{\prime \prime}, \mathbf{r}\right)
$$

for arbitrary $L^{\prime \prime}<L$ in the finite volume $\mathcal{U}$. Thus Eqs. (B1), (B2), (B5) and (B7), (2.12) yield

$$
\mathcal{F}_{G}=-n \int d \mathbf{r}\left[\mathcal{M}(\mathbf{r})+\frac{1}{2} \mathcal{S M}^{2}(\mathbf{r})\right],
$$

and the free energy cost of immersing the particle has the form of Eq. (2.13).

\section{APPENDIX C: SOLUTION OF SELF-CONSISTENT EQUATIONS}

In order to solve the system of Eqs. (2.8)-(2.12), we introduce the Laplace transform of the partition function $Z(L, \mathbf{r})[$ see Eq. (A1)]

$$
\chi(t ; \mathbf{r})=\mathcal{L} Z(L, \mathbf{r}) .
$$


If we now set

$$
\widetilde{\rho}=r_{\perp} / \sqrt{L}, \quad \tau=L t+\mathcal{S}
$$

and

$$
\tilde{\chi}_{\tau}(\widetilde{\rho})=\chi(t ; \mathbf{r}) / L
$$

then Eqs. (2.8) and (2.10) lead to

$$
-\widetilde{\chi}_{\tau}^{\prime \prime}(\widetilde{\rho})-\frac{d_{\perp}-1}{\widetilde{\rho}} \widetilde{\chi}_{\tau}^{\prime}(\widetilde{\rho})+[\tau+\delta \tilde{\mathcal{V}}(\widetilde{\rho})] \widetilde{\chi}_{\tau}(\widetilde{\rho})=1,
$$

with

$$
\delta \tilde{\mathcal{V}}(\widetilde{\rho})=\mathcal{S}\left[-1+\mathcal{M}\left(r_{\perp}\right)\right] .
$$

According to Eq. (2.12),

$$
\mathcal{M}\left(r_{\perp}\right)=\int_{\gamma} \frac{d \tau}{2 \pi i} e^{\tau} \tilde{\chi}_{\tau}^{2}(\widetilde{\rho}),
$$

where the integration path $\gamma$ is parallel to the imaginary axis and to the right of all singularities of the integrand. The boundary condition Eq. (2.11) now reads

$$
\tilde{\chi}_{\tau}(\tilde{\rho} \rightarrow R / \sqrt{L}) \rightarrow 0,
$$

and the bulk limit is given by

$$
\lim _{\widetilde{\rho} \rightarrow \infty} \tilde{\chi}_{\tau}(\widetilde{\rho})=\frac{1}{\tau} .
$$

To solve Eq. (C4) with the conditions Eqs. (C7), (C8), we assume that the range of the potential $\delta \widetilde{\mathcal{V}}$ is finite, i.e., there exists $^{51}$ a $\widetilde{\rho}_{0}$ with $\delta \widetilde{\mathcal{V}}(\widetilde{\rho}) \equiv 0$ for $\widetilde{\rho} \geqslant \widetilde{\rho}_{0}$. Thus, in the range $\tilde{\rho} \geqslant \widetilde{\rho}_{0}$ the relevant linear differential equation is

$$
-\widetilde{\chi}_{\tau}^{\prime \prime}(\widetilde{\rho})-\frac{d_{\perp}-1}{\widetilde{\rho}} \widetilde{\chi}_{\tau}^{\prime}(\widetilde{\rho})+\tau \widetilde{\chi}_{\tau}(\widetilde{\rho})=1
$$

with the boundary condition Eq. (C8), whereas in the range $\widetilde{\rho}_{s} \equiv R / \sqrt{L} \leqslant \widetilde{\rho}<\widetilde{\rho}_{0}$ we numerically solve the initial value problems

$$
\begin{aligned}
& -g_{\tau}^{\prime \prime}(\widetilde{\rho})-\frac{d_{\perp}-1}{\widetilde{\rho}} g_{\tau}^{\prime}(\widetilde{\rho})+[\tau+\delta \widetilde{\mathcal{V}}(\widetilde{\rho})] g_{\tau}(\widetilde{\rho})=1, \\
& g_{\tau}\left(\widetilde{\rho}_{s}\right)=0, \quad g_{\tau}^{\prime}\left(\widetilde{\rho}_{s}\right)=0
\end{aligned}
$$

and

$$
\begin{aligned}
& -h_{\tau}^{\prime \prime}(\widetilde{\rho})-\frac{d_{\perp}-1}{\widetilde{\rho}} h_{\tau}^{\prime}(\widetilde{\rho})+[\tau+\delta \widetilde{\mathcal{V}}(\widetilde{\rho})] h_{\tau}(\widetilde{\rho})=0, \\
& h_{\tau}\left(\widetilde{\rho}_{S}\right)=0, \quad h_{\tau}^{\prime}\left(\widetilde{\rho}_{S}\right)=1 .
\end{aligned}
$$

The solution to Eq. (C4) is given by

$$
\begin{aligned}
\tilde{\chi}_{\tau}(\widetilde{\boldsymbol{\rho}})= & \left(g_{\tau}(\widetilde{\boldsymbol{\rho}})+c_{1} h_{\tau}(\widetilde{\boldsymbol{\rho}}), \quad \frac{1}{\tau}+c_{2} \widetilde{\rho}^{-\alpha} K_{\alpha}(\widetilde{\rho} \sqrt{\tau})\right) \\
& \text { for }\left(R / \sqrt{L} \leqslant \widetilde{\rho}<\widetilde{\rho}_{0}, \quad \widetilde{\rho}_{0}<\widetilde{\rho}\right),
\end{aligned}
$$

where $\alpha=\left(d_{\perp}-2\right) / 2$ and $K_{\alpha}$ is a modified Bessel function. The constants $c_{1}$ and $c_{2}$ are calculated from the continuity condition of $\tilde{\chi}_{\tau}$ and $\tilde{\chi}_{\tau}^{\prime}$ at $\widetilde{\rho}=\widetilde{\rho}_{0}$.

Thus after choosing a starting ${ }^{52}$ potential $\delta \widetilde{\mathcal{V}}_{0}(\widetilde{\rho})$, we calculate with $(\mathrm{C} 10)-(\mathrm{C} 12)$ a solution $\tilde{\chi}_{\tau}^{0}$, which by means of Eqs. (C6) and (C5) yields a new potential $\delta \widetilde{\mathcal{V}}_{1}(\widetilde{\rho})$. Solving the problem Eqs. $(\mathrm{C} 10)-(\mathrm{C} 12)$ again with $\delta \tilde{\mathcal{V}}(\widetilde{\rho})$ replaced by $\delta \widetilde{\mathcal{V}}_{1}(\widetilde{\rho})$ and following the same steps leads to a potential $\delta \widetilde{\mathcal{V}}_{2}(\widetilde{\rho})$ and so on. The sequence $\delta \widetilde{\mathcal{V}}_{i}(\widetilde{\rho})$ then converges to the self-consistent potential $\delta \widetilde{\mathcal{V}}(\widetilde{\rho})$, which yields the monomer density $\mathcal{M}\left(r_{\perp}\right)$ directly from Eq. (C5).

\section{APPENDIX D: EXPANSION FOR SMALL OVERLAP}

In this section we calculate analytically the scaling functions $g(\mathcal{S})$ and $h(\mathcal{S})$ of the surface tension and the coefficient of spontaneous curvature in the dilute limit $\mathcal{S} \ll 1$. First we expand the density profile $\mathcal{M}(\mathbf{r})$ in powers of $\mathcal{S}$

$$
\mathcal{M}(\mathbf{r})=\mathcal{M}^{[0]}(\mathbf{r})+\mathcal{S}\left[\mathcal{M}^{[0]}(\mathbf{r})-\mathcal{M}^{[1]}(\mathbf{r})\right],
$$

where $\mathcal{M}^{[0]}$ is the monomer density for ideal chains. The form of $\mathcal{M}^{[1]}$ follows from Eqs. (A25), (B4) and Fig. 19 and satisfies the identity

$$
\int_{r_{\perp}>R} d \mathbf{r}_{\perp}\left\{\mathcal{M}^{[1]}(\mathbf{r})-\left[\mathcal{M}^{[0]}(\mathbf{r})\right]^{2}\right\}=0
$$

[see Eq. (B7)]. Inserting Eqs. (D1) and (D2) into Eq. (2.13), one finds

$$
\begin{aligned}
\frac{F / V_{\|}}{k_{B} T}= & n\left\{V_{\perp}+\int_{r_{\perp}>R} d \mathbf{r}_{\perp}\left[1-\mathcal{M}^{[0]}(\mathbf{r})\right]\right\} \\
& +\frac{\mathcal{S}}{2} n\left\{V_{\perp}+\int_{r_{\perp}>R} d \mathbf{r}_{\perp}\left[1-\mathcal{M}^{[0]}(\mathbf{r})\right]^{2}\right\}+O\left(\mathcal{S}^{2}\right) .
\end{aligned}
$$

To obtain the coefficient of spontaneous curvature, we expand to first order in $1 / R$. The expansion for $\mathcal{M}^{[0]}$ is given by

$$
\begin{aligned}
\mathcal{M}^{[0]}(\mathbf{r})= & \mathcal{M}_{\mathrm{pw}}^{[0]}\left(r_{\perp}-R\right) \\
& +\frac{d_{\perp}-1}{2} \frac{r_{\perp}-R}{R} \delta \mathcal{M}^{[0]}\left(r_{\perp}-R\right),
\end{aligned}
$$

with

$$
\begin{aligned}
& \mathcal{M}_{\mathrm{pw}}^{[0]}(z)=1-8 \mathrm{i}^{2} \operatorname{erfc}(y)+4 \mathrm{i}^{2} \operatorname{erfc}(2 y), \\
& \delta \mathcal{M}^{[0]}(z)=8 \mathrm{i}^{2} \operatorname{erfc}(y)-8 \mathrm{i}^{2} \operatorname{erfc}(2 y),
\end{aligned}
$$

where $\mathrm{i}^{2}$ erfc is the second iterated complementary error function and

$$
y=\frac{z}{2 \sqrt{L}}=\frac{1}{\sqrt{2}} \frac{z}{\mathcal{R}_{x}} .
$$

After inserting Eq. (D4) into Eq. (D3) and setting $r_{\perp}$ $=R+z$, comparison with Eq. (2.28) yields

$\frac{\sigma}{n k_{B} T}=\int_{0}^{\infty} d z\left\{1-\mathcal{M}_{\mathrm{pw}}^{[0]}(z)+\frac{\mathcal{S}}{2}\left[1-\mathcal{M}_{\mathrm{pw}}^{[0]}(z)\right]^{2}\right\}+O\left(\mathcal{S}^{2}\right)$

and 


$$
\begin{aligned}
\frac{\kappa}{n k_{B} T}= & 2 \int_{0}^{\infty} d z z\left(1-\mathcal{M}_{\mathrm{pw}}^{[0]}(z)-\frac{1}{2} \delta \mathcal{M}^{[0]}(z)\right) \\
& +\mathcal{S}\left\{\int _ { 0 } ^ { \infty } d z z [ 1 - \mathcal { M } _ { \mathrm { pw } } ^ { [ 0 ] } ( z ) ] \left[1-\mathcal{M}_{\mathrm{pw}}^{[0]}(z)\right.\right. \\
& \left.\left.-\delta \mathcal{M}^{[0]}(z)\right]\right\}+O\left(\mathcal{S}^{2}\right) .
\end{aligned}
$$

All the integrals can be calculated analytically and give the results in Eqs. (2.32) and (2.35), respectively.

\section{APPENDIX E: THE SEMI-DILUTE LIMIT}

The self-consistent mean-field procedure in Eqs. (2.8)(2.12) simplifies considerably in the semidilute limit $\mathcal{S} \rightarrow \infty$. Due to "ground-state dominance" ${ }^{6}$ the $L^{\prime}$-dependence in

$$
Z_{L^{\prime}}(\mathbf{r}) \rightarrow e^{-L^{\prime} \mathcal{S} / L} \psi(\mathbf{r})
$$

factors, i.e., $\psi$ is independent of $L^{\prime}$, and Eq. (2.12) leads to

$$
\mathcal{M}(\mathbf{r})=\psi^{2}(\mathbf{r}) .
$$

Thus $\psi$ vanishes at the particle surface and tends to 1 far away from the particle. Substituting Eq. (E1) into the diffusion-type equation (2.8) and using Eqs. (2.9) and (E2) yields $^{53}$

$$
\frac{L}{\mathcal{S}} \Delta_{\mathbf{r}} \psi(\mathbf{r})=-\psi(\mathbf{r})+\psi^{3}(\mathbf{r}) .
$$

Note that the characteristic length $\sqrt{L / \mathcal{S}}$ in Eq. (E3) is of the order of the correlation or screening length. ${ }^{31,54}$

Equation (E3) can be used to calculate the bulknormalized density $\mathcal{M}$ for a planar wall and for a generalized cylinder of large radius $R$. In this case,

$$
\begin{aligned}
\Delta_{\mathbf{r}} \psi\left(r_{\perp}\right) & =\left(\frac{d^{2}}{d r_{\perp}^{2}}+\frac{d_{\perp}-1}{r_{\perp}} \frac{d}{d r_{\perp}}\right) \psi\left(r_{\perp}\right) \\
& \rightarrow\left(\frac{d^{2}}{d r_{\perp}^{2}}+\frac{d_{\perp}-1}{R} \frac{d}{d r_{\perp}}\right) \psi\left(r_{\perp}\right),
\end{aligned}
$$

which implies the small-curvature expansion,

$$
\psi(\mathbf{r})=\psi_{\mathrm{pw}}(x)+\delta \psi(x),
$$

with $\delta \psi=O\left(\left(\mathcal{R}_{x} / \sqrt{\mathcal{S}}\right) / R\right)$. Here

$$
\psi_{\mathrm{pw}}(x)=\operatorname{Th} x,
$$

with

$$
x=\left(r_{\perp}-R\right) \sqrt{\mathcal{S} /(2 L)}=\frac{r_{\perp}-R}{\mathcal{R}_{x}} \sqrt{\mathcal{S}},
$$

is the solution of

$$
\frac{1}{2} \frac{d^{2}}{d x^{2}} \psi_{\mathrm{pw}}=-\psi_{\mathrm{pw}}+\psi_{\mathrm{pw}}^{3}
$$

and determines the density profile $\mathcal{M}_{\mathrm{pw}}=\psi_{\mathrm{pw}}^{2}$ near a planar wall. Collecting the terms of order $\left(\mathcal{R}_{x} / \sqrt{\mathcal{S}}\right) / R$ in Eqs. (E3)(E5) leads to the linear inhomogeneous equation,

$$
\left(\frac{d^{2}}{d x^{2}}+2-6 \psi_{\mathrm{pw}}^{2}\right) \delta \psi(x)=-\frac{\mathcal{R}_{x} / \sqrt{\mathcal{S}}}{R}\left(d_{\perp}-1\right) \frac{d}{d x} \psi_{\mathrm{pw}},
$$

for $\delta \psi$. The solution of Eq. (E9) which vanishes both for $x$ $\rightarrow 0$ and $x \rightarrow \infty$ is given by

$$
\delta \psi(x)=\frac{\mathcal{R}_{x} / \sqrt{\mathcal{S}}}{R}\left(d_{\perp}-1\right) \Psi(x),
$$

where

$$
\Psi(x)=\frac{1}{6}\left[\frac{3}{2} \frac{x}{\operatorname{Cosh}^{2} x}+\frac{2}{\operatorname{Cosh}^{2} x}+\frac{3}{2} \operatorname{Th} x-\frac{3}{2}-\frac{1}{2} e^{-2 x}\right] .
$$

The behavior of $\mathcal{M}$ near the surface follows from substituting Eqs. (E5), (E6), and (E10), (E11) into Eq. (E2). This yields

$$
\mathcal{M} \rightarrow\left(\frac{r_{\perp}-R}{\mathcal{R}_{x}}\right)^{2} \mathcal{S}\left[1+\frac{\mathcal{R}_{x} / \sqrt{\mathcal{S}}}{R} \frac{4}{3}\left(d_{\perp}-1\right)\right],
$$

which is consistent with the density-pressure prediction in Eq. (2.37).

Inserting Eqs. (E2), (E5), (E6), (E10), (E11) for $\mathcal{M}$ into Eqs. (2.13) and (2.19) leads to the free energy in the semidilute limit. Note that the second term in the integrand of Eq. (2.13) dominates in the semidilute limit $\mathcal{S} \rightarrow \infty$. On comparing ${ }^{55}$ the $1 / R$ expansion of the integral in Eqs. (2.13), (2.19),

$$
\begin{aligned}
& S_{\perp} \frac{\mathcal{R}_{x} \sqrt{\mathcal{S}}}{2} \int_{0}^{\infty} d x\left[1+\frac{\mathcal{R}_{x} / \sqrt{\mathcal{S}}}{R} x\right]^{d_{\perp}-1}\left[1-\psi_{\mathrm{pw}}^{4}-4 \psi_{\mathrm{pw}}^{3} \delta \psi\right] \\
& \rightarrow S_{\perp}\left[\sigma+\frac{d_{\perp}-1}{2} \frac{\kappa}{R}\right] /\left(n k_{B} T\right),
\end{aligned}
$$

with the small curvature expansion Eq. (2.28), one finds the surface tension,

$$
\frac{\sigma}{n k_{B} T}=\frac{\mathcal{R}_{x} \sqrt{\mathcal{S}}}{2} \int_{0}^{\infty} d x\left[1-\psi_{\mathrm{pw}}^{4}(x)\right],
$$

and the coefficient of spontaneous curvature,

$$
\frac{\kappa}{n k_{B} T}=\mathcal{R}_{x}^{2} \int_{0}^{\infty} d x\left\{x\left[1-\psi_{\mathrm{pw}}^{4}(x)\right]-4 \psi_{\mathrm{pw}}^{3}(x) \Psi(x)\right\} .
$$

Inserting $\psi_{\mathrm{pw}}$ and $\Psi$ from Eqs. (E6) and (E11) and evaluating the integrals leads to the semidilute results in Eqs. (2.32) and (2.35).

After the discussion of a cylinder with large radius $\xi$ $\ll R$ let us now turn to the density profile around a thin cylinder with $R \ll \xi$. While for $R, r_{\perp} \ll \xi$ Eq. (1.4) applies we find for $R \ll r_{\perp}, \xi$ from Eq. (2.41),

$$
\mathcal{M} \rightarrow 1-2 \frac{R}{r_{\perp}} e^{-r_{\perp} / \xi}
$$

with $^{54}$

$$
\xi=\sqrt{L /(2 \mathcal{S})}=\mathcal{R}_{x} /(2 \sqrt{\mathcal{S}}) .
$$


Here we have taken into account that $\mathcal{R}_{x}$ is much larger than $r_{\perp}$ and $\xi$ and have expanded the Debye function in Eq. (2.41) for large argument.

Finally we consider the scaling behavior in the semidilute limit for $R / \xi$ arbitrary. From Eq. (E3) one finds for the density profile,

$$
\mathcal{M}\left(r_{\perp}\right)=\widetilde{m}\left(\frac{r_{\perp}}{R}, \frac{R}{\xi}\right) .
$$

For the free energy cost Eqs. (2.13), (2.19) in the semidilute limit,

$$
\frac{F / V_{\|}}{n k_{B} T} \rightarrow \frac{\mathcal{S}}{2}\left\{V_{\perp}+\int d^{3} \mathbf{r}_{\perp}\left[1-\mathcal{M}^{2}\left(r_{\perp}\right)\right]\right\},
$$

one finds the scaling forms,

$$
\frac{F / V_{\|}}{n k_{B} T}=\mathcal{S} R^{3} \widetilde{Y}(R / \xi)=R \mathcal{R}_{x}^{2} Y(R / \xi)
$$

and Eqs. (1.5) and (2.28), (2.31)-(2.35) lead to the limiting behaviors,

$$
\begin{aligned}
Y(y) \rightarrow & {\left[2 \pi, \frac{\pi}{6} y^{2}+\frac{4 \pi}{3} y+\frac{4 \pi}{3}(4 \ln 2-1)\right] } \\
& \text { for } y \rightarrow[0, \infty] .
\end{aligned}
$$

${ }^{1}$ R. Tuinier et al., Phys. Rev. E 60, 848 (1999).

${ }^{2}$ J. Janzen and D. E. Brooks, Clin. Hemorheol. 9, 695 (1989).

${ }^{3}$ A. M. Kulkarni, A. P. Chatterjee, K. S. Schweizer, and C. F. Zukoski, Phys. Rev. Lett. 83, 4554 (1999); J. Phys.: Condens. Matter 12, A301 (2000).

${ }^{4}$ A. P. Minton, Curr. Opin. in Biotechnology 8, 65 (1997).

${ }^{5}$ P. R. Wills et al., Biophys. Chem. 57, 37 (1995).

${ }^{6}$ P. G. de Gennes, Scaling Concepts in Polymer Physics (Cornell University, Ithaca, 1979).

${ }^{7}$ J. des Cloizeaux and G. Jannink, Polymers in Solution (Clarendon, Oxford, 1990).

${ }^{8}$ L. Schaefer, Excluded Volume Effects in Polymer Solutions (Springer, Berlin, 1999).

${ }^{9}$ E. Eisenriegler, Polymers Near Surfaces (World Scientific, Singapore, 1993).

${ }^{10}$ For $R \ll r_{\perp} \ll \mathcal{R}_{x}, \xi$, the profile $\mathcal{M}_{\mathrm{S}}$ deviates from its bulk value 1 according to the power-law $1-\mathcal{M}_{\mathrm{s}} \propto\left(R / r_{\perp}\right)^{d_{\perp}-(1 / \nu)}$, provided $d_{\perp}>1 / \nu$. Here $d_{\perp}=d$ or $d_{\perp}=d-1$ for a sphere or a cylinder, respectively, and $\nu$ is the Flory exponent (Refs. 6-8).

${ }^{11}$ T. M. Birshtein and O. V. Borisov, Polymer 32, 916 (1991).

${ }^{12}$ T. Odijk, Macromolecules 29, 1842 (1996); J. Chem. Phys. 106, 3402 (1996); Physica A 278, 347 (2000).

${ }^{13}$ E. Eisenriegler, A. Hanke, and S. Dietrich, Phys. Rev. E 54, 1134 (1996).

${ }^{14}$ E. Eisenriegler, J. Physics: Condens. Matter 12, A227 (2000).

${ }^{15}$ K. M. Jansons and C. G. Phillips, J. Colloid Interface Sci. 137, 75 (1990).

${ }^{16}$ A. Hanke, E. Eisenriegler, and S. Dietrich, Phys. Rev. E 59, 6853 (1999).

${ }^{17}$ K. R. Myers and K. F. Freed, J. Chem. Phys. 98, 2437 (1993).

${ }^{18}$ P. G. de Gennes, C.R. Acad. Sc. Paris B 288, 359 (1979).

${ }^{19}$ J. F. Joanny, L. Leibler, and P. G. de Gennes, J. Polym. Sci., Polym. Phys. Ed. 17, 1073 (1979)

${ }^{20}$ E. Eisenriegler, Phys. Rev. E 55, 3116 (1997).

${ }^{21}$ Unlike the "wall-theorem" for a hard sphere fluid involving the density right at the wall, i.e., at the smallest possible distance, the density-pressure identity for long flexible polymers involves the density at distances from the wall or particle surface which are in the scaling regime and much larger than the monomer size, although much smaller than $R$ and the mesoscopic polymer lengths.
${ }^{22}$ The spatial dimension $d=4$ is of special interest, since (Refs. 6-8) crossings of random-walklike chains are irrelevant for $d>4$. A perturbative treatment of the excluded-volume interaction between chain segments or "beads" is possible for $d / 4$.

${ }^{23}$ E. Eisenriegler, in Field Theoretical Tools in Polymer- and ParticlePhysics, edited by H. Meyer-Ortmanns and A. Klümper, Lecture Notes in Physics, 508 (Springer, Berlin, 1998)

${ }^{24}$ A. P. Chatterjee and K. S. Schweizer, J. Chem. Phys. 109, 10477 (1998).

${ }^{25}$ See the discussion in the Appendix of Ref. 24.

${ }^{26}$ M. Fuchs and K. S. Schweizer, Europhys. Lett. 51, 621 (2000).

${ }^{27}$ The density profile of ideal chains outside a cylinder in four dimensions equals (Ref. 35) the profile outside a sphere in three dimensions and can be found in Eq. (3.4) of Ref. 13.

${ }^{28}$ Compare the Note added in proof in Ref. 14 and the discussion in Sec. IIF below.

${ }^{29}$ W. Helfrich, Z. Naturforsch. C 28, 693 (1973).

${ }^{30}$ Note from Eqs. (2.2), (2.3), and (2.4) that (Ref. 8) the two different limits $\alpha_{0} s_{d}^{(x)}$ and $\alpha_{\infty}\left(s_{d}^{(x)}\right)^{1 /(d \nu-1)}$ of $\left[\Pi /\left(k_{B} T n\right)\right]-1$ for $s_{d}^{(x)} \rightarrow 0$ and $s_{d}^{(x)} \rightarrow \infty$ become equal for small $\epsilon$. Equation (2.7) is consistent with the leading term $\epsilon / 8$ in the $\epsilon$-expansion (Ref. 8) of the "interpenetration-ratio" $\psi_{d}$ $=A_{2} /\left(\sqrt{2 \pi} \mathcal{R}_{x}\right)^{d}$.

${ }^{31}$ Another simple quantity marking the crossover is the density correlation length $\xi_{D}$ defined as the square root of the second moment of the density correlation function. In the mean-field approximation $\xi_{D}^{2}=(d / 3) \mathcal{R}_{x}^{2} /(1$ $+\mathcal{S})$.

${ }^{32}$ Readers familiar with mean-field theories for polymers on a lattice will recognize Eqs. (2.8) -(2.12) as the continuum counterparts of relations on a lattice. Compare the discussion near Eqs. (4.1.23), (4.4.20), and (4.1.32) in: G. J. Fleer, M. A. Cohen Stuart, J. M. H. M. Scheutjens, T. Cosgrove, and B. Vincent, Polymers at Interfaces (Chapman and Hall, London, 1993). The present continuum theory does not use a "ground-state approximation" (Ref. 6) and provides a mean-field description for depletion in a polymer system of low density and long flexible chains. For a curved colloidal surface it is more natural and easier to treat than a lattice model.

${ }^{33}$ For small $R$ the power-law $R^{d_{\perp}-1 / \nu}$ appears both in the solvation free energy and in the density profile (Refs. 10, 13, 16). The value of the exponent for a sphere in three dimensions, $d_{\perp}-1 / \nu \approx 1.33$, is approximated more closely by the starting ( $\epsilon=0)$ value 1 of route Eq. (2.14) than by the starting value 2 of the route with $d_{\|}=0$.

${ }^{34}$ For $\epsilon \backslash 0$ the quantity $\mathcal{S}$ rather than $s_{d}^{(x)}$ is the physically relevant variable. See Eq. (2.5) and the discussion below this equation.

${ }^{35}$ For the generalized cylinder the mean-field partition function $Z\left(L^{\prime}, \mathbf{r}\right)$ $\equiv Z\left(L^{\prime}, \mathbf{r}_{\|}, \mathbf{r}_{\perp}\right)$ in Eq. (2.8) factors into two functions $Z_{\|}\left(L^{\prime}, \mathbf{r}_{\|}\right)=1$ and $Z_{\perp}\left(L^{\prime}, r_{\perp}\right)=\left[Z\left(L^{\prime}, \mathbf{r}\right)\right]_{d \rightarrow d_{\perp},|\mathbf{r}|=r_{\perp}}$ which are independent of $\mathbf{r}_{\|}$and $d_{\|}$. For finite $\epsilon>0$ the mean-field approximation and independence of $d_{\|}$do not apply.

${ }^{36}$ The scaling forms Eqs. (2.15) and (2.20) for $\mathcal{M}$ and $F$ hold not only for $d \nearrow 4$ but also for $d<4$, provided the leading order expressions for $\mathcal{R}_{x}$ from Eq. (2.1) and for $n^{*}$ from Eq. (2.7) are replaced by $\mathcal{R}_{\mathrm{EE}}(d) / \sqrt{d}$, with $\mathcal{R}_{\mathrm{EE}}(d)$ the rms end-to-end distance of an isolated self-avoiding chain, and by the reciprocal virial coefficient $\left[A_{2}(d)\right]^{-1}$ of self- and mutually avoiding chains in $d<4$. However, the scaling functions $m$ and $f$ for $d$ $<4$ are different from the mean-field scaling functions.

${ }^{37}$ The result Eq. (2.30) could also be obtained by comparing Eq. (2.13) with (2.28) for the case of a plate, in which $d_{\perp}=1$ and $S_{\perp}=2$.

${ }^{38}$ Mean-field results for small overlap and in the semidilute limit are discussed in Appendices D and E, respectively.

${ }^{39}$ E. Eisenriegler, J. Chem. Phys. 79, 1052 (1983).

${ }^{40} \mathrm{~J}$. van der Gucht, N. A. M. Besseling, J. van Male, and M. A. Cohen Stuart, J. Chem. Phys. 113, 2886 (2000).

${ }^{41}$ P. G. Bolhuis, A. A. Louis, J. P. Hansen, and E. J. Meijer, J. Chem. Phys. 114, 4296 (2001).

${ }^{42}$ The tree approximation of the density cumulant in the bulk polymer solution as given by the Fourier integral in Eq. (2.41) has a negative minimum value. This minimum corresponds to the maximum in $\mathcal{M}$ shown in Fig. 16.

${ }^{43}$ Our quantities $b, u_{r}, L_{r}$ are denoted by $l^{4}\left|\hat{b}_{2}\right|, u^{R}, L^{R}$ in Ref. 9, and our $\frac{2}{3} u_{r}$ equals the quantity $u$ in Ref. 8. Our choice $f=(4 \pi)^{(d / 2)}-2$ for the parameter $f$ in Ref. 9 corresponds to $b_{u}=1$ in Ref. 8 .

${ }^{44}$ The left hand side of Eq. (3.10) is basically (the mean-field expression, 
Ref. 31, of) the inverse square of the dimensionless renormalized density correlation length of the noncritical system.

${ }^{45}$ D. R. Nelson, Phys. Rev. B 14, 1123 (1976).

${ }^{46} \rho_{0}$ and $a / \zeta_{0}$ with $a$ from Eq. (3.15) correspond to the quantities $n_{0}$ and $c_{0}$ in Ref. 8.

${ }^{47}$ While $\rho_{0}$ follows from the "interpenetration ratio" $\psi^{*}=0.245$ in the dilute limit behavior $\Pi /\left(n k_{B} T\right) \rightarrow 1+\frac{1}{2}(4 \pi)^{3 / 2} \psi^{*} s$, the constant $\zeta_{0}$ follows from the value $\mathcal{A}_{\infty / 2}=1.098$ in three dimensions of the amplitude ratio $\mathcal{A}_{\infty / 2}=\left(\lim _{s \rightarrow \infty} s^{-1}[\mathcal{P}(s)]^{\nu d-1}\right) /\left(\lim _{s \rightarrow 0} s^{-1} \mathcal{P}(s)\right) \quad$ where $\quad \mathcal{P}(s)=-1$ $+\Pi /\left(n k_{B} T\right)$

${ }^{48}$ We use curly brackets for Ginzburg-Landau averages and reserve angular brackets for averages over chain configurations.

${ }^{49}$ For example, in $d=3$ dimensions this can be checked for the complete set $f\left(\Omega_{S}\right)=e^{i m \varphi_{S}} P_{l}^{m}\left(\cos \vartheta_{S}\right)$ of spherical harmonics by using the "addition theorem" to express $C_{l}^{(1 / 2)}\left(\cos \vartheta_{\mathbf{r}_{S}, \mathbf{r}_{A}}\right)=P_{l}\left(\cos \vartheta_{\mathbf{r}_{S}, \mathbf{r}_{A}}\right)$ in terms of a sum of products of spherical harmonics.

${ }^{50}$ We use the fact that the limit $\mathbf{r} \rightarrow \mathbf{r}_{S}$ in Eq. (A23) can be interchanged with the integrations in the tree diagrams of Eq. (A24). This interchange does not hold in general for the loop-integrations in nontree diagrams. See the discussion of short distance expansions in Ref. 9.

${ }^{51}$ We always made sure that $\widetilde{\rho}_{0}$ was chosen large enough.

${ }^{52}$ We always started with $\delta \widetilde{\mathcal{V}}_{0}(\rho) \equiv 0$, so that the solution $\widetilde{\chi}_{\tau}^{0}$ is the well known analytical result for the dilute limit.

${ }^{53}$ For a polymer solution with an embedded particle inside a large but finite volume $\mathcal{U}$ the grand canonical free energy per $k_{B} T$ Eq. (B8) in the semidilute limit, $-n(\mathcal{S} / 2) \int d \mathbf{r} \mathcal{M}^{2}(\mathbf{r})$, can be viewed (Ref. 6) as the minimal value of the Ginzburg-Landau type functional $2 n \mathcal{S} \int d \mathbf{r}\left[(L /(2 \mathcal{S}))(\nabla \psi)^{2}-\frac{1}{2} \psi^{2}+\frac{1}{4} \psi^{4}\right]$.

${ }^{54}$ The density bulk correlation function of a semidilute polymer solution in $d$ dimensions decays for $\xi \ll r \ll \mathcal{R}_{x}$ in the mean-field approximation as $\propto r^{-(d-1) / 2} e^{-r / \xi}$ where $\xi$ is given by Eq. (E17).

${ }^{55}$ Our result confirms the form Eq. (2.28) of the Helfrich expansion for generalized cylinders with arbitrary $d_{\perp} \leqslant d$. 